\title{
Entropy bound and unitarity of scattering amplitudes
}

\author{
Gia Dvali \\ Arnold Sommerfeld Center, Ludwig-Maximilians-Universität, \\ Theresienstraße 37, 80333 München, Germany \\ Max-Planck-Institut für Physik, \\ Föhringer Ring 6, 80805 München, Germany \\ E-mail: dvali.physics@gmail.com
}

ABSTRACT: We establish that unitarity of scattering amplitudes imposes universal entropy bounds. The maximal entropy of a self-sustained quantum field object of radius $R$ is equal to its surface area and at the same time to the inverse running coupling $\alpha$ evaluated at the scale $R$. The saturation of these entropy bounds is in one-to-one correspondence with the non-perturbative saturation of unitarity by $2 \rightarrow N$ particle scattering amplitudes at the point of optimal truncation. These bounds are more stringent than Bekenstein's bound and in a consistent theory all three get saturated simultaneously. This is true for all known entropy-saturating objects such as solitons, instantons, baryons, oscillons, black holes or simply lumps of classical fields. We refer to these collectively as saturons and show that in renormalizable theories they behave in all other respects like black holes. Finally, it is argued that the confinement in $\mathrm{SU}(N)$ gauge theory can be understood as a direct consequence of the entropy bounds and unitarity.

Keywords: Effective Field Theories, Nonperturbative Effects, Solitons Monopoles and Instantons, Global Symmetries

ArXiv ePrint: 2003.05546 


\section{Contents}

1 Introduction 2

2 Entropy of a lump 5

2.1 Lump as multi-particle state 5

$\begin{array}{lll}2.2 & \text { Inverse-coupling }=\text { area-law }=\text { unitarity } & 7\end{array}$

3 Connection with amplitudes $\quad 9$

3.1 Exponential suppression of individual $n$-particle micro-states 11

$\begin{array}{lll}3.2 & \text { Entropy enhancement } & 12\end{array}$

4 Entropic meaning of confinement $\quad \mathbf{1 5}$

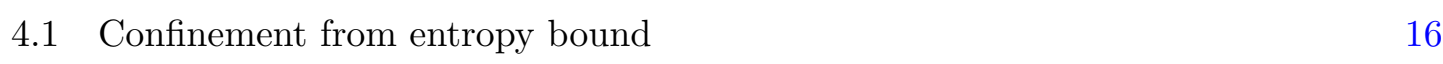

$\begin{array}{lll}4.2 \text { Baryons } & 19\end{array}$

5 Scanning the cross section 20

$\begin{array}{lll}5.1 \text { Scanning } \lambda_{c} & 20\end{array}$

$\begin{array}{ll}5.2 \text { Scanning } \lambda_{t}(q) & 21\end{array}$

6 A model of saturon as vacuum bubble $\quad 23$

6.1 The model 23

$\begin{array}{lll}\text { 6.1.1 Small bubbles as saturons } & 26\end{array}$

$\begin{array}{lll}\text { 6.1.2 Suppression of large bubbles } & 27\end{array}$

6.1.3 Superiority of area-law and inverse-coupling bounds 28

7 Black holes as saturons $\quad 30$

$\begin{array}{lll}7.1 & \text { Similarities in entropy } & 30\end{array}$

7.2 Decay and life-time 31

7.3 Infomation horizon and time-scales 33

$\begin{array}{lll}7.4 & \text { Scattering amplitudes } & 34\end{array}$

8 Saturons and classicalization $\quad 35$

$\begin{array}{lll}9 & \text { Gravitational species bound } & 37\end{array}$

$\begin{array}{ll}10 \text { Outlook } & 39\end{array}$

A Argument from effective $S$-matrix $\quad 41$ 


\section{Introduction}

The purpose of this paper is to show that unitarity of scattering amplitudes imposes the following universal non-perturbative upper bounds on the entropy of the system.

- The area-law entropy bound:

The maximal entropy of any self-sustained quantum field theoretic object localized within a sphere of radius $R$ is equal to the area of the sphere measured in units of the relevant Goldstone decay constant $f$ :

$$
S_{\max }=\frac{\text { Area }}{f^{-2}} .
$$

- The inverse-coupling entropy bound:

The maximal entropy of any self-sustained quantum field theoretic object localized within a sphere of radius $R$ is equal to the inverse of the running coupling $\alpha(q)$ of the relevant long-range interaction evaluated at the scale of momentum-transfer $q=\frac{1}{R}$.

$$
S_{\max }=\frac{1}{\alpha} .
$$

We shall argue that a violation of the above bounds leads to a non-perturbative violation of unitarity.

The foundation for this connection was already laid down in previous articles $[1,2]$. Namely, it was observed there that entropy of a self-sustained field theoretic object such as soliton or a baryon of mass $M$ and radius $R$ saturates (1.1) and (1.2) simultaneously with Bekenstein's entropy bound [3],

$$
S_{\max }=2 \pi M R .
$$

This happens exclusively when the theory saturates unitarity. That is, the following relations emerge.

First, the maximal entropy is always equal to the surface area of the object, measured in units of the decay constant $f$ of the Goldstone field, as given by (1.1). This Goldstone mode is universally present due to the fact that any localized field configuration breaks spontaneously set of symmetries, which obviously include Poincare translations. However, there also emerge the Goldstone mode(s) corresponding to the breaking of internal symmetries. This shall become clear below.

Secondly, the same maximal entropy is equal to an inverse of the running coupling $\alpha$ evaluated at the scale $q=1 / R$, as described by (1.2). Of course, what matters is the interaction with the range that covers $R$. Note, when the scale $R$ separates two different regimes, the equation (1.2) must be satisfied from both sides. For example, in case of a baryon of size $R$, it is satisfied both by gluons and by pions. 
Thus, in $[1,2]$ the entropy bound attained by various objects was observed to satisfy the following relation,

$$
S_{\max }=M R=\frac{1}{\alpha}=\frac{\text { Area }}{f^{-2}} .
$$

(Throughout the paper, the order-one numeric factors shall be explicitly shown only when they are important.)

From here, the following natural questions emerge:

- Are the three bounds (1.1), (1.2) and (1.3) equivalent?

- And if not, which of them is more fundamental?

The main goal of the present paper is to understand the independent fundamental meanings of the area-law (1.1) and the inverse-coupling (1.2) entropy bounds and their connection to unitarity. First, we shall achieve this by analysing scattering amplitudes. Secondly, we shall construct explicit renormalizable theories in which the saturation of the three different bounds can be monitored in various parameter regimes.

The first part of our message is to establish an universal connection between the bounds (1.1) and (1.2) and scattering amplitudes. Namely, there exists a one-to-one correspondence between the saturation of (1.1) and (1.2) by an arbitrary field theoretic entity - irrespectively whether of Lorentzian or Euclidean signature - and non-perturbative saturation of unitarity by a set of $2 \rightarrow n$ amplitudes with $n=\frac{1}{\alpha}$ at momentum-transfer $q=\frac{1}{R}$. This saturation is non-perturbative and cannot be removed by resummation.

Surprisingly, the bounds (1.1) and (1.2) turn out to be more stringent than the Bekenstein bound (1.3). As we shall see, in some situations these bounds can be violated even when the Bekenstein bound (1.3) is still respected. Such examples are immediately killed by unitarity. This is because the bounds (1.1) and (1.2) control the saturation of unitarity by the scattering amplitudes. On the other hand, in all examples known to us, the saturation of the bounds (1.1) and (1.2) automatically leads to the saturation of the bound (1.3). Therefore, the saturation of the two former bounds appears to provide the necessary and sufficient condition for reaching the maximal entropy permitted by the consistency of the theory. Thus, in a consistent theory at the saturation point the entropy satisfies the triple equation (1.4).

A natural physical interpretation of the above amplitudes at the saturation point is that they describe a creation of $n$-particle composite object. This object saturates the entropy bounds (1.1) and (1.2) and correspondingly satisfies (1.4). We shall refer to such objects as saturons. The process thus schematically can be presented as a creation of a classical object in a two-particle scattering,

$$
2 \rightarrow n=\text { saturon }
$$

The reason why the cross-section of such a process is not exponentially suppressed is that the saturon exhausts all possible final states in the given kinematic regime. So in this sense saturons effectively provide the mechanism of classicalization of the scattering amplitude [4]. Of course, explaining how this happens is one of the central points of our paper. 
However, the above should not create a false impression that it is easy to produce a saturon in a high energy scattering experiment. Although, at its mass-threshold the saturon's cross section saturates unitarity at the expense of its maximal entropy, there is a price to pay. It comes in form of a very narrow $\left(\frac{\Delta E}{E} \sim \alpha\right)$ "window of opportunity" for the choice of the center of mass energy $E$ of the initial state. Due to this, in order for saturons to play a role in UV-completion of the theory, they must fill an almost continuous mass spectrum. This is possible if the theory possess a non-trivial fixed point. In such a case, saturons can play an interesting role both in UV-completion as well as in collider phenomenology.

From the point of view of fundamental physics, one of the implications of the bounds (1.1) and (1.2) is to put phenomena such as confinement in a new light. Namely, it was already suggested in [1] that confinement in $\mathrm{SU}(N)$ gauge theory can be viewed as a built-in defence mechanism against violations of the entropy bounds. Here, we provide more evidence for this. Namely, we consider an example presented in [2] of $\mathrm{SU}(N)$ gauge theory in which the entropy bounds (1.1) and (1.2) are saturated by an instanton. We show that this saturation is mapped on the saturation of unitarity by a set $2 \rightarrow N$-gluon amplitudes. From here it is evident that in order not to violate these bounds the theory must become confining at large distances. That is, without confinement there is no visible mechanism that would prevent such a violation at some IR scale.

Analogously, when quarks are included, the theory resists against violation of the bounds (1.1) and (1.2) by baryons. Namely, a baryon saturates both entropy bounds when the number of the quark flavors is of the same order as the number of colors. The baryon entropy in this limit is given by its area measured in units of the pion decay constant [1]. Simultaneously, the $2 \rightarrow N$ pion cross section saturates unitarity. In this case, the violation of the bounds (1.1) and (1.2) would render the theory asymptotically not free and thus inconsistent in UV.

Finally, an important message of the present paper is the understanding of black holes and the saturons of renormalizable theories as the representatives of the same saturon family. In order to make the parallels maximally sharp, we construct an explicit renormalizable theory which contains saturons. These are the solitonic vacuum bubbles. In the interior of the bubble $N$ distinct gapless Goldstone modes are localized. These gapless modes endow the bubble with a large micro-state entropy. We then show that at the point when the bubble saturates the entropy bounds (1.1) and (1.2), the corresponding amplitudes saturate unitarity. So, the bubble becomes a saturon. At this point, all its properties become identical to the known properties of a black hole.

For example, both the renormalizable saturon and a black hole obey the relation (1.4). Here, we must remember that for a black hole $f=M_{P}$, where $M_{P}$ is the Planck mass. Indeed, first, $M_{P}$ represents the graviton decay constant. Secondly, the Goldstone boson of a translation symmetry that is spontaneously broken by a black hole, is the graviton itself. This immediately shows that the famous Bekenstein-Hawking entropy [5] satisfies the relation (1.4). Next, just like a black hole, in the semi-classical limit $(N=\infty)$ the non-gravitational saturon possesses an information horizon. It emits particles in a way that is strikingly similar to Hawking's emission. In particular, the information stored in the saturon's interior cannot be decoded by analysing the emitted radiation. In contrast, 
for finite $N$, the saturon bubble does release information albeit very slowly. The timescales are identical to the ones that are commonly attributed to a black hole. Finally, both a black hole and a non-gravitational saturon saturate unitarity in respective multiparticle scatterings. This features are universal and independent on a particular nature of a saturon. So they are shared by saturons in other renormalizable theories.

The natural interpretation of the above striking connection is that a black hole of size $R$ represents a saturated state of the soft gravitons of wavelength $R$, as this has been long advocated by the black hole $N$-portrait $[6,7]$. In this paper the relation (1.2) for black holes has already been noticed. This relation was used there as a guiding principle for establishing the similarity between black holes and other saturated states such as BoseEinstein condensates at criticality. The present paper reinforces this view.

\section{Entropy of a lump}

Before moving to amplitudes, we shall establish mapping between localized field theory configurations with Lorentzian signature, such as solitons or lumps, and $n$-particle states. We explain why for such objects the bound (1.4) holds.

\subsection{Lump as multi-particle state}

Consider degrees of freedom described by creation/annihilation operators $\hat{a}_{j}(\vec{k})^{\dagger}, \hat{a}_{j}(\vec{k})$. Here the label $\vec{k}$ refers to momentum, whereas $j=1, \ldots, N$ is the species label describing different spin and internal states. For example, $j$ can denote sets of color or flavor indexes. We shall assume that operators obey the standard bosonic commutation relations, $\left[\hat{a}_{i}(\vec{k}), \hat{a}_{j}\left(\overrightarrow{k^{\prime}}\right)^{\dagger}\right]=\delta_{i j} \delta_{\vec{k} \vec{k}^{\prime}},\left[\hat{a}_{i}(\vec{k}), \hat{a}_{j}\left(\overrightarrow{k^{\prime}}\right)\right]=0$. That is, $\hat{a}_{j}(\vec{k})$ represent different physical modes of a bosonic quantum field $\hat{\phi}_{j}$,

$$
\hat{\phi}_{j}=\sum_{\vec{k}} \frac{1}{\sqrt{\omega_{\vec{k}}}}\left(\mathrm{e}^{i \vec{k} \vec{x}} \hat{a}_{j}(\vec{k})+\mathrm{e}^{-i \vec{k} \vec{x}} \hat{a}_{j}(\vec{k})^{\dagger}\right) .
$$

This field can either be fundamental or represent an effective description of some more fundamental theory. For example, $\hat{\phi}_{j}$ may represent the low energy fluctuations of quarkanti-quark condensate in QCD. We shall also assume that the effective Hamiltonian is invariant under an internal symmetry $\mathcal{G}$ that acts on the label $j$. Again, this symmetry can be either emergent or be fundamental.

Next, we shall denote by $\alpha$ the strength of an effective four-boson interaction,

$$
\alpha\left(\hat{\phi}_{i} \hat{\phi}_{i}\right)\left(\hat{\phi}_{j} \hat{\phi}_{j}\right)+\ldots,
$$

The above notation is highly schematic. Throughout the paper we shall assume the coupling $\alpha$ to be weak. In fact, defining the analog of the 't Hooft coupling,

$$
\lambda_{t} \equiv \alpha N
$$

our methods shall be most reliable in the limit,

$$
\alpha \rightarrow 0, \lambda_{t}=\text { finite } .
$$

This is analogous to 't Hooft's limit [8]. 
Now, we wish to focus on states in which modes of certain momentum $\vec{k}$ are highly occupied

$$
|n\rangle_{\text {micro }}=\prod_{j=1}^{N} \frac{\left(\hat{a}_{j}(\vec{k})^{\dagger}\right)^{n_{j}}}{\sqrt{n_{j} !}}|0\rangle,
$$

where $n$ refers to a total occupation number,

$$
n=\sum_{j=1}^{N} n_{j} .
$$

This number will be assumed to be very large. We shall refer to such states as micro-states. This is because they are distinguished solely by different microscopic distributions of the total occupation number $n$ among the $j$-species. And, in the limit (2.4) they become indistinguishable. Such states therefore describe different micro-states of the same macro-state $|n\rangle$.

Obviously, in such a state the wave-functions of $n$ bosonic modes overlap, similarly to what happens in Bose-Einstein condensates. It is therefore useful to introduce a concept of the collective coupling defined as,

$$
\lambda_{c} \equiv \alpha n
$$

Again, our analysis is most reliable in the following double-scaling limit,

$$
\alpha \rightarrow 0, \lambda_{c}=\text { finite }
$$

Despite the superficial similarity between $\lambda_{c}$ and $\lambda_{t}$, the two couplings are physically very different. It is enough to note that the 't Hooft coupling $\lambda_{t}$ is a parameter of the theory, whereas the collective coupling $\lambda_{c}$ is a parameter of the state. Despite this difference, as we shall see, the two couplings become comparable and critical on the states that saturate the entropy bounds (1.1), (1.2) and (1.4).

Now, using the number-eigenstates (2.5), we can form the coherent states that represent classical field-configurations localized within certain characteristic radius $R$. They have a form,

$$
|s o l\rangle=\mathrm{e}^{\sum_{\vec{k}} \sum_{j=1}^{N} \sqrt{n_{j}(\vec{k})}\left(\hat{a}_{j}(\vec{k})^{\dagger}-\hat{a}(\vec{k})_{j}\right)}|0\rangle
$$

with

$$
\sum_{j=1}^{N} \sum_{\vec{k}} n_{j}(\vec{k})=n \gg 1,
$$

where $n_{j}(\vec{k})$-s are sharply peaked around the characteristic momentum $|\vec{k}| \sim \frac{1}{R} \equiv q$. Obviously, the corresponding classical field is described by the expectation value,

$$
\phi_{j}=\left\langle\operatorname{sol}\left|\hat{\phi}_{j}\right| s o l\right\rangle,
$$

of the quantum field. We shall refer to such a state as a lump or a soliton. Of course, such a field configuration in general depends on time. It evolves both classically as well as quantum mechanically. Since the quantum coupling $\alpha$ is weak, the classical (mean field) 
evolution is valid for sufficiently long time. We are interested in field configurations that spread-out from the initial localization on time-scales $t \gg R$. This constraint does not apply to internal oscillations of the lump, as long as they stay localized within the radius $R$. At weak coupling, this requirement is satisfied by most of the self-sustained solitonic configurations. The condition for self-sustainability will be derived below.

Under such conditions, the localized classical field configuration, $\phi_{\text {sol }}$, can be treated as $n$-particle state of characteristic momenta $\sim q=1 / R$, each contributing $\sim q$ into the energy of the lump. The total energy therefore is,

$$
E \sim \frac{n}{R}
$$

Now, assuming that at distances $\sim R$ the interaction is attractive, let us estimate the number of constituents required for creating a self-sustained bound-state. This can be done by balancing the kinetick energy of each quantum, $E_{k i n} \sim \frac{1}{R}$, against the attractive potential energy from the rest. The latter goes as $E_{p o t} \sim \frac{\alpha n}{R}$. This gives the equilibrium condition,

$$
\text { Critical balance: } \lambda_{c}=\alpha n \sim 1 \text {. }
$$

We thus learn that the self-sustained configuration is reached when the collective coupling $\lambda_{c}$ is order one, or equivalently, when $n \sim \frac{1}{\alpha}$. Inserting this relation in (2.12), we get for the energy of the bound-state,

$$
E_{\mathrm{sol}} \sim \frac{q}{\alpha} \sim \frac{1}{\alpha R}
$$

The latter is a well-known relation between the energy of a soliton and its size.

Note, of corse, in general, in a self-sustained bound-state, the particles do not strictly satisfy the dispersion relation $\omega_{\vec{k}}=\sqrt{m^{2}+|\vec{k}|^{2}}$ with $m$ being a mass of a free particle. That is, the operators $\hat{a}_{j}(\vec{k})$ of the bound-state are related with analogous operators of free asymptotic quanta by a non-trivial Bogoliubov transformation. However, in the regime (2.13) at large- $n$ this difference is unimportant for our purposes. In this regime, the self-sustained states can consistently be mapped on the scattering amplitudes.

\subsection{Inverse-coupling $=$ area-law $=$ unitarity}

We now wish to derive the entropy of the lump and establish for which values of parameters it saturates the bounds (1.1) and (1.2). For this, we need to count the number of degenerate micro-states. As already noted, the states (2.5) (or (2.9)) represent particular microstates belonging to one and the same classical macro state. This is due to the following reasons. First, such states form large representations under the symmetry $\mathcal{G}$ that acts on the label $j$. Secondly, because the quantum coupling $\alpha$ is vanishingly small, the timescale for differentiating between individual "colors" or "flavors" is macroscopically large. Correspondingly, such states are classically indistinguishable.

Thus, the number of degenerate micro-states is given by the dimensionality of representation that they form under the symmetry group $\mathcal{G}$. This dimensionality is easy to estimate. For example, in the simplest case of a symmetric wave-function, $n_{j}$-s can assume arbitrary values subject to the constraint (2.6) (or (2.10)). Therefore, the number of 
micro-states is given by the following binomial coefficient:

$$
n_{\mathrm{st}} \simeq\left(\begin{array}{c}
n+N \\
N
\end{array}\right)=c_{N}\left(\left(1+\frac{\lambda_{t}}{\lambda_{c}}\right)^{\lambda_{c}}\left(1+\frac{\lambda_{c}}{\lambda_{t}}\right)^{\lambda_{t}}\right)^{\frac{1}{\alpha}},
$$

where we have used the Stirling approximation for large $N=\frac{\lambda_{t}}{\alpha}$ and $n=\frac{\lambda_{c}}{\alpha}$. Notice, the coefficient $c_{N} \simeq \sqrt{\frac{1}{2 \pi}\left(N^{-1}+n^{-1}\right)}$ can be replaced by one without any loss of information. This is the benefit of working at large $N$ and at the saturation point. Since we shall take advantage of this fact throughout the paper, we shall explain it briefly here.

The trick is that the saturation values of $\lambda_{t}$ and $\lambda_{c}$ are determined by matching the quantities that are exponentially sensitive to $N$ and $n$ (equivalently, to $\alpha^{-1}$ ). Therefore, the coefficients such as $c_{N}$, that exhibit power-law dependence on $N$ and $n$, play essentially no role in it. Such quantities correct the saturation value of $\lambda_{t}$ only by the amount $\sim \frac{\ln (N)}{N}$ which vanishes in the 't Hooft limit (2.4). Therefore, all such coefficients can be set equal to one without compromising our analysis.

Then, taking the collective coupling at the critical value $\lambda_{c}=1$, the number of states becomes

$$
n_{\mathrm{st}} \simeq\left(\left(1+\lambda_{t}\right)\left(1+\frac{1}{\lambda_{t}}\right)^{\lambda_{t}}\right)^{\frac{1}{\alpha}}
$$

The corresponding entropy of the soliton/lump is,

$$
S=\ln \left(n_{\mathrm{st}}\right) \simeq \frac{1}{\alpha} \ln \left(\left(1+\lambda_{t}\right)\left(1+\frac{1}{\lambda_{t}}\right)^{\lambda_{t}}\right) .
$$

This entropy saturates the bound (1.2) for,

$$
\text { Entropy saturation : } \lambda_{t} \simeq 0.54 \text {. }
$$

Of course, what matters is that the critical 't Hooft coupling is order one. However, the above numerical value obtained for $\lambda_{c}=1$ will be useful as a reference point for the later estimates. As a consistency check, notice that the actual value of $c_{N}$ corrects (2.18) by the amount $\sim \frac{\ln (N)}{N}$ and is negligible.

Thus, we discover that the $n$-particle state, describing a self-sustained classical soliton/lump, saturates the entropy bound (1.2) when the 't Hooft and collective couplings are both of order one,

$$
\lambda_{c} \sim \lambda_{t} \sim 1
$$

As already pointed out in $[1,2]$, through the above equation, the saturation of entropy is correlated with the saturation of unitarity. The depth of this correlation will be explored throughout the paper.

Now, following $[1,2]$, it is easy to see that at the saturation point the entropy becomes equal to an area of the soliton/lump in units of the Goldstone decay constant $f$. Let us therefore determine the latter. The localized classical field configuration $\phi$ breaks spontaneously both the Poincare symmetries such as space-translations as well as the internal 
symmetries. The order parameter of breaking the translation invariance is $\nabla \phi \sim \frac{1}{R^{2} \sqrt{\alpha}}$. Consequently, the decay constant of the corresponding Goldstone fields is

$$
f=\frac{1}{R \sqrt{\alpha}}=\frac{\sqrt{N}}{R} .
$$

Notice, the above expression also determines the decay constants of the Goldstone modes of spontaneously broken internal symmetries. These are the symmetries under which the lump/soliton transforms non-trivially. Previously, they were schematically denoted by $\mathcal{G}$. The explicit examples will be constructed below.

It is now obvious that the entropy (2.17) at the saturation point of the bound (1.2) can be written as,

$$
S_{\max }=\frac{1}{\alpha}=(R f)^{2}=\frac{\text { Area }}{f^{-2}} .
$$

Notice, the equation (2.20) relates the Goldstone decay constant $f$ with the running coupling $\alpha$ evaluated at the scale $1 / R$. All the scale dependence, such as the logarithmic running of the coupling with the scale, must already be included in $\alpha$ entering both in equation (2.20) as well as in equation (1.2). Correspondingly, the equation (2.21) includes no additional log factors.

Thus, the areal-law bound (1.1) is saturated simultaneously with (1.2). As already stressed in $[1,2]$, this is strikingly similar to a black hole entropy with the role of the Planck mass played by $f$.

Thus, we discover that the saturation of the inverse-coupling entropy bound (1.2) takes place together with the saturation of the area-law bound (1.1). It is very important that this happens when the value of the 't Hooft coupling is order one (2.19). This fact is the key for connecting the saturation of the above entropy bounds to unitarity.

Notice, the saturation of the bounds (1.1) and (1.2) implies the saturation of the Bekenstein bound (1.3). This can be seen easily by inserting (2.14) in the Bekenstein formula (1.3). We get

$$
S_{\mathrm{Bek}}=E R=\frac{1}{\alpha} .
$$

Thus, a self-sustained quantum field theoretic system with a single characteristic localization scale $R$ satisfies (1.4). This is exactly the result obtained in [1,2].

However, the converse is not true in general. That is, a satisfaction of the Bekenstein bound (1.3) does not guarantee the satisfaction of the bounds (1.1) or (1.2). However, such examples violate unitarity and, therefore, are inconsistent. Thus, the areal-law and the inverse-coupling bounds turn out to be more restrictive than the Bekenstein bound. We conclude that in a consistent theory all three bounds must be saturated together (1.4). In all examples known to us this proves to be the case.

\section{Connection with amplitudes}

The equation (2.17) tells us that the classical lump saturates the entropy bounds (1.1) and (1.2) and satisfies (1.4) when the 't Hooft coupling $\lambda_{t}$ equals to the critical value (2.19) 

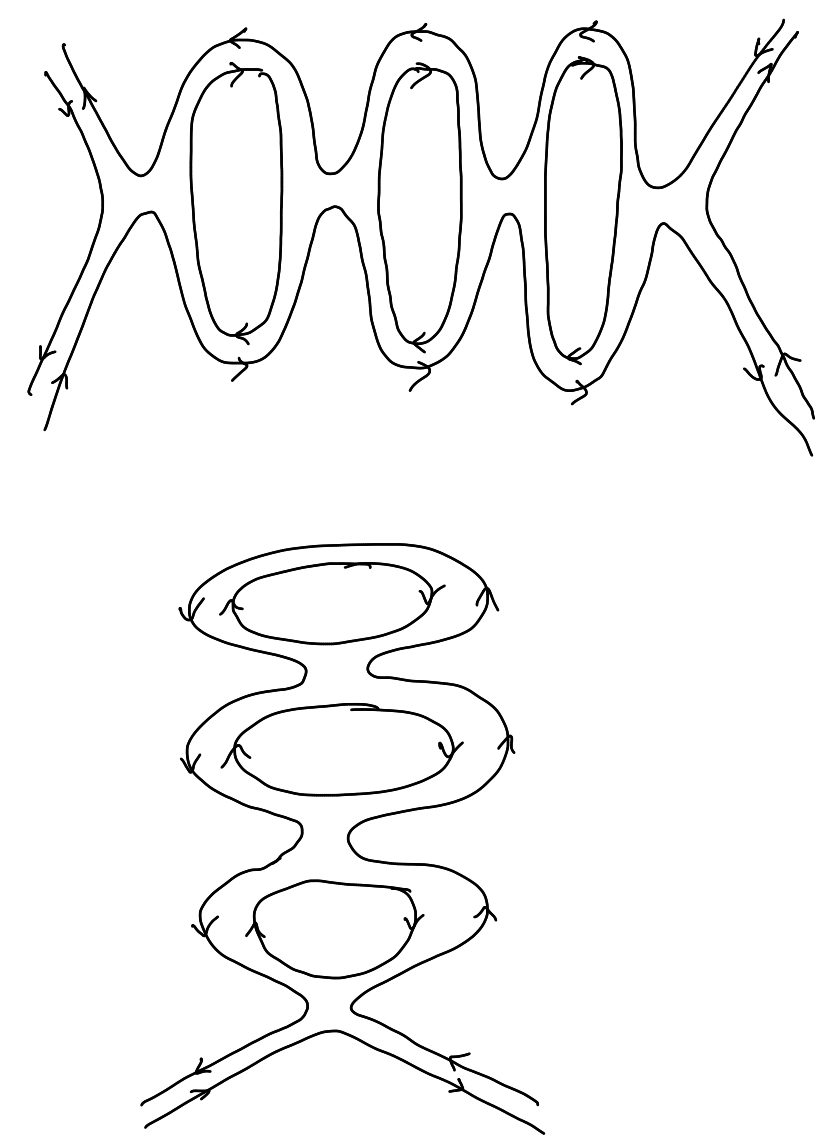

Figure 1. A typical diagram that violates perturbative expansion in powers $\lambda_{t}$ in 't Hooft's double line notations. Each extra bubble brings an additional factor $\lambda_{t}$.

(or more explicitly, (2.18)). We now wish to connect this phenomenon to the saturation of unitarity by certain scattering amplitudes.

As the first step, let us have a closer look at the nature of would-be violation of unitarity at strong 't Hooft coupling. The first place where this violation is manifest is the loop expansion. An example is given by bubble diagrams depicted on figure 1 .

Since the addition of each bubble carries a factor $\sim \lambda_{t}$, the expansion breaks down for large $\lambda_{t}$. From the first glance, one would think that such breakdown of unitarity is not fundamental and can be bypassed by re-summation. While the bubble diagrams are resummable, the question is whether this procedure renders the saturation of unitarity unphysical. We shall argue that this is not the case.

The important processes to look at are the multi-particle amplitudes of the sort $2 \rightarrow n$, in which the final $n$-particle state has the form (2.5). We wish to show that such processes saturate unitarity whenever the inverse-coupling entropy bound (1.2) is saturated by the final state. The same applies to the area-law bound (1.1). This saturation is physical and cannot be removed by resummation.

However, in order to avoid confusion, we must keep a clear separation between the following two summations. 
- The first one is the resummation of all Feynman diagrams that contribute to the transition amplitude into a specific $n$-particle micro-state (2.5).

- The second is the summation - in the cross section - over all micro-states (2.5) that belong to the same classical macro-state.

We focus on the first one first.

\subsection{Exponential suppression of individual $n$-particle micro-states}

In order to clearly distinguish the false saturation of unitarity from the real one, consider first a theory in which the final state particles do not transform under any large symmetry group $\mathcal{G}$. In this case, we can simply temporarily forget about the label $j$ in the final state. Of course, we still assume that the four-point coupling $\alpha$ is weak. In such a theory, we look for a transition from an initial 2-particle state into a state (2.5). The latter contains a high occupation number $n$ with some characteristic momentum $q=1 / R$. As already discussed, the proper coherent superposition of such states (2.9) can be viewed as a lump or a solitonic wave of a classical field.

It is well-accepted (see, [15]-[23]) that the cross-section for such a process must be exponentially suppressed. This is true, despite the fact that the multiplicity of contributing Feynman diagrams grows factorially with $n$ already at the tree-level [24, 25]. Namely, at large $n$ the perturbative cross-section behaves as,

$$
\sigma_{2 \rightarrow n}=c_{n} n ! \alpha^{n},
$$

where only the leading factorial and exponential scalings in $n$ are displayed explicitly. All the standard integration, not connected with the $\mathcal{G}$-degeneracy of the final state, is included in the prefactor $c_{n}$ which has proper dimensionality. In particular, if theory is gapless, $c_{n}$ will include the standard infrared dressing due to emission of infinitely-soft quanta.

As explained previously, since the prefactor $c_{n}$ exhibits a power-law dependence on $n$, it is unimportant for physics close to saturation point at large $n$. Therefore, as previously, we set all such coefficients equal to one. The maximal error we commit with this setting is $\sim \frac{\ln (n)}{n}$.

The factorial growth of the perturbative cross section (3.1) creates a false impression that at large $n$ unitarity can be saturated (or even violated) at weak coupling $\alpha$ by a single final micro-state. Or to put it differently, a classical object can saturate unitarity without summation over final states of internal degeneracy $\mathcal{G}$. This is not true, since for $n>\alpha^{-1}$ the growth of (3.1) is unphysical and cannot be trusted. The reason is that the perturbative expansion in $\alpha$ breaks down beyond this point.

Indeed, thinking of cross section in terms of expansion in series of $\alpha$, we must stop as soon as $\sigma_{2 \rightarrow n}$ reaches the minimum in $n$. This happens at $n=\alpha^{-1}$, i.e., for the critical value of the collective coupling,

$$
\text { Optimal truncation: } \lambda_{c}=1 \text {. }
$$

Hence, we shall adopt this value of the collective coupling as the point of optimal truncation of series in $\alpha$. It is highly instructive that this optimal value of $\lambda_{c}$ coincides with its critical 
value obtained by the self-sustainability condition (2.13). This is no accident and it reveals how the information about the non-perturbative solitonic state penetrates in the realm of scattering amplitudes.

Now, using Stirling approximation, it is easy to see that for the critical value (3.2) the cross section (3.1) is exponentially suppressed,

$$
\sigma_{2 \rightarrow n}=\mathrm{e}^{-n}=\mathrm{e}^{-\frac{1}{\alpha}}
$$

This suppression represents an embodiment of the difficulty of producing a classical object in a two-particle scattering process.

From (3.2), it is clear that the expression (3.1) can only be trusted for $n \leqslant \alpha^{-1}$. Beyond this point it must be abandoned and non-perturbative methods must be used. This non-perturbative analysis [15]-[21] confirm the exponential suppression of transitions to states with high occupation number $n$.

However, for self-sufficiency, in the appendix we present a refined version of a short-cut non-perturbative argument of [22]. It shows that for $n \gg \alpha^{-1}$ the cross-section of any given $n$-particle state (2.5) is suppressed as

$$
\sigma_{2 \rightarrow n} \lesssim n ! n^{-n} \sim \mathrm{e}^{-n}
$$

Notice, this is only a consistency upper bound and in reality the suppression could be much stronger. However, the above upper bound is sufficient for our considerations.

\subsection{Entropy enhancement}

We thus adopt a physically justified picture that, in the absence of large internal degeneracy $\mathcal{G}$, the cross section of producing a high-occupation number state is exponentially suppressed, as given by (3.3) and (3.4).

However, in the presence of a large internal degeneracy group $\mathcal{G}$, a new twist appears. The theory now can give rise to classical objects that saturate entropy bound (1.2). From quantum field theory perspective they represent the high occupation number states with exponential degeneracy $n_{\text {st }}=\mathrm{e}^{\frac{1}{\alpha}}$.

In such a case, while the exponential suppression of the properly resummed individual processes (3.4) continues to hold, the number of processes that contribute into creation of a given classical object is exponentially large. This number is equal to the number of micro-states $n_{\text {st }}$ that belong to the same classical macro-state. The total cross section of production of the classical object is thus obtained by summing over all such micro-states,

$$
\sigma=\sum_{\text {micr.st }}^{n_{\mathrm{st}}} \sigma_{2 \rightarrow n} .
$$

Notice, here and below the notation $\sigma$ refers exclusively to the part of the cross-section that describes a creation of a given classical object.

We are now ready to understand the fundamental meaning of the inverse coupling bound (1.2) in terms of the unitarity of the scattering amplitudes. For this, let us first note 
that for large $n$ the summation over the micro-states in (3.5) reduces to a multiplication by the micro-state degeneracy factor $n_{\text {st }}=\mathrm{e}^{S}$,

$$
\sigma=\sigma_{2 \rightarrow n} \mathrm{e}^{S}
$$

Using (3.3), at the point of optimal truncation, $\lambda_{c}=1$, this becomes,

$$
\sigma=\mathrm{e}^{-\frac{1}{\alpha}+S} .
$$

From this expression it is clear that the cross section (3.7) saturates/violates unitarity whenever the entropy $S$ saturates/violates the bound (1.2). That is, the number of microstates $n_{\text {st }}$ compensates the exponential suppression of individual amplitudes precisely when the classical object saturates the inverse-coupling entropy bound (1.2). At this point $\sigma$ becomes an all-inclusive cross-section and the corresponding classical object becomes a saturon.

The term saturation of unitarity must be understood in the standard way that the cross section becomes maximal compatible with unitarity. In particular, it will saturate the Froissart bound for a given theory.

The above phenomenon comes from an additional enhancement of the cross section due to an internal degeneracy $\mathcal{G}$. This degeneracy is responsible for the maximal entropy of the classical final-state. This saturation cannot be removed by any resummation. As discussed above, this effect is very different from a "false" saturation of unitarity due to factorial multiplicity of Feynman diagrams of individual amplitudes.

It is useful to translate the unitarity bound in terms of 't Hooft coupling. For this, we again focus at the optimal truncation point $n=\frac{1}{\alpha}$. Then, the individual cross sections are given by (3.3) and the total one is given by (3.7). Expressing the entropy $S$ through (2.17), we can rewrite $(3.7)$ as

$$
\sigma=\left(\left(\left(1+\lambda_{t}\right) \frac{1}{\mathrm{e}}\right)^{\frac{1}{\lambda_{t}}}\left(1+\frac{1}{\lambda_{t}}\right)\right)^{N}
$$

The critical value of $\lambda_{t}$ for which the above cross section saturates unitarity is,

$$
\text { Unitarity saturation: } \lambda_{t} \simeq 0.54 \text {. }
$$

Of course, $\lambda_{t}$ here must be understood as the running 't Hooft coupling evaluated at the scale $q$. As it is clear from (2.18), the exact same value also saturates the entropy bound (1.2).

As we can see from (3.9), the bound is saturated for the critical value of the 't Hooft coupling that is order one. This is typical and may raise some concerns. One may worry that at the saturation point the perturbative expansion in $\lambda_{t}$ may not be reliable (although not out of question for (3.9)).

However, the fact that at the saturation point $\lambda_{t}$ is "borderline", as opposed to being much larger than one, gives an important advantage. It allows to unambiguously capture the tendency of saturation while approaching the saturation point from the domain of 
weak 't Hooft coupling. In other words, we clearly see a synchronized growth of the entropy and of the cross section when we increase $\lambda_{t}$ while it is still smaller than one and, correspondingly, falls within a perturbative control. Extrapolating this tendency, we reach the conclusion that, for certain critical value $\lambda_{t} \sim 1$, the saturation of unitarity by the cross section does take place. In such a case, the corresponding value of $S$ should be taken as the field theoretic bound on the entropy. Using the expression (3.7) as the guideline, the corresponding bound on entropy is given by (1.2).

We thus see that the saturation of the cross section by a classical object gives the inverse-coupling entropy bound (1.2). The object therefore represents a saturon. Its mass and the size are uniquely determined as,

$$
\text { Saturon mass: } M \sim \frac{q}{\alpha} \sim \frac{1}{\alpha R}
$$

and

$$
\text { Saturon size: } R \sim \frac{1}{q},
$$

where $q$ is the scale at which the running 't Hooft coupling reaches the critical value (3.9).

It is clear that simultaneously the area law bound (1.1) is also saturated. Indeed, the saturon state breaks spontaneously both the space translations as well as the internal symmetry that acts on index $j$. The decay constant of the resulting Goldstone modes is $f=\frac{\sqrt{n}}{q}=\frac{q}{\sqrt{\alpha}}$. It is then obvious from (3.11) that the final state entropy $S=\frac{1}{\alpha}$ that saturates the inverse-coupling bound is equal to the area of the saturon in units of the Goldstone decay constant $f$.

Finally, it is clear from (3.10) and (3.11) that the Bekenstein (1.3) bound is also saturated. The saturon, therefore, saturates the combined bound (1.4).

The physical meaning of the above finding is pretty transparent. When we form an $n$-particle state in a 2-particle collision, we are effectively forming a classical object. The formation probability is exponentially suppressed by $\mathrm{e}^{-n}$. This suppression is confirmed both by the previous analysis [15]-[21] as well as by the non-perturbative argument of [22] presented in the appendix.

However, when the classical object saturates the entropy bound (1.2), the novelty appears. Now, the theory contains exponentially large number of copies of the same classical object. I say "copies" because classically they are indistinguishable from one another. Indeed, a classical observer, Alice, cannot resolve the "flavor" index $j$ since the coupling vanishes as $\alpha \sim \frac{\lambda_{t}}{N} \sim \frac{\lambda_{c}}{n}$. Rather, Alice is only sensitive to the effects controlled by 't Hooft and collective couplings. That is, Alice cannot tell the difference between the states with different $j$-content, as long as the total occupation number $n$ is large.

Correspondingly, the production of any of these micro-states in a scattering experiment will be interpreted by Alice as the production of one and the same classical state. Now, while each particular transition matrix element is exponentially suppressed, all of them will contribute to the Alice's classical count. Once the number of micro-states reaches the critical value, this classical object saturates the scattering cross-section.

It is clear that this effect cannot be removed by any further re-summation. Indeed, the resummation helps to compute the correct cross sections of the individual $2 \rightarrow n$ 

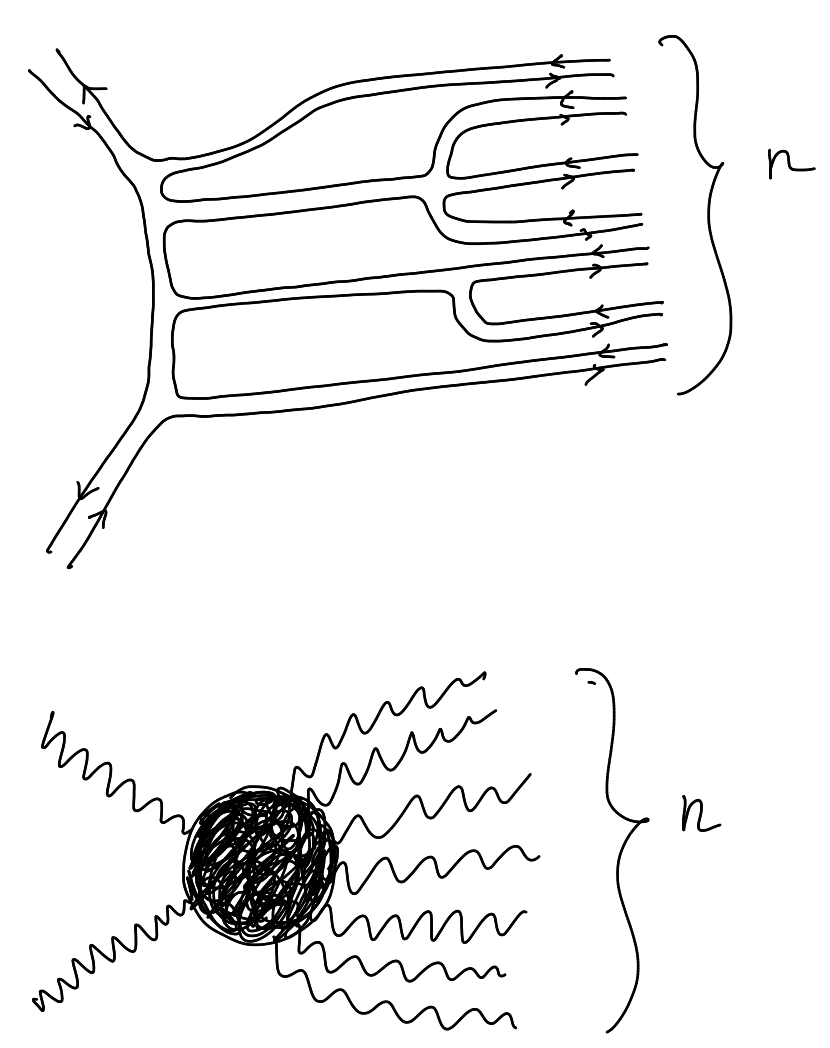

Figure 2. A $2 \rightarrow n$ process and an example of diagram in 't Hooft double-line notation contributing in it.

physical processes. They come out exponentially suppressed (3.3)-(3.4), as they should. At the same time, the resummation cannot reduce the number of physically distinct final states. As a result, no matter how suppressed are the individual processes, the suppression gets compensated by the multiplicity of final micro-states when the corresponding microstate entropy saturates the bound (1.2). This is a fully non-perturbative phenomenon highlighting a deep connection between entropy and unitarity.

\section{Entropic meaning of confinement}

One remarkable thing in connection between entropy and unitarity is that the saturation is fully controlled by 't Hooft and collective couplings, $\lambda_{t}, \lambda_{c}$. At the same time, the quantum coupling $\alpha$ can be arbitrarily weak. It is fair to ask:

What happens if we try to deform the theory and push the state beyond the saturation point?

This can be done by fixing the collective coupling at the critical value $\lambda_{c}=1$ while increasing the 't Hooft coupling. From (2.15) it is clear that for $\lambda_{t} \rightarrow \infty$ the number of micro-states increases as,

$$
n_{\mathrm{st}} \simeq\left(\mathrm{e} \lambda_{t}\right)^{\frac{1}{\alpha}} .
$$


Correspondingly, the entropy of the macro-state increases as,

$$
S \simeq \frac{1}{\alpha}\left(1+\ln \left(\lambda_{t}\right)\right)
$$

Consequently, for $\lambda_{t} \gg 1$ the bound (1.2) is violated. Simultaneously, the cross section (3.8) diverges as,

$$
\sigma \simeq\left(\lambda_{t}\right)^{\frac{1}{\alpha}}
$$

and violates unitarity. Obviously, in a consistent theory this cannot happen. What is the lesson that we are learning from here?

As a minimalistic move, we must adopt the saturation value as a consistency upper bound on 't Hooft coupling. The precise value depends on the representation content under the symmetry group $\mathcal{G}$ but in general is order one.

Yet, the story must be more profound. It would be somewhat counter-intuitive if a theory allows us to cross into a dangerous domain without a prior warning. Of course, one can say that violation of unitarity by a multi-particle state is a clear warning sign. However, we expect that a consistent theory does not stop here. Instead, it must block the entrance into the dangerous domain of the parameter space dynamically.

Therefore, we would like to ask whether a consistent theory possesses a built-in mechanism that prevents such deformations from happening. We shall now argue that confinement in $\mathrm{SU}(N)$ gauge theory represents such a preventive mechanism agains the violations of the entropy and unitarity bounds. This idea has already been put forward in [1] and we shall now elaborate on it.

\subsection{Confinement from entropy bound}

As an illustrative example, we consider a $\mathrm{SU}(N)$ Yang-Mills gauge theory with no fermions. As it is well-known, this theory is asymptotically free, with the running gauge coupling $\alpha(q)$ becoming weak at short distances. We shall define the 't Hooft coupling $\lambda_{t}$ as before (2.3) and shall be working in 't Hooft's limit (2.4). Obviously, in this limit QCD scale $\Lambda_{Q C D}$ is kept fixed.

Now, as shown in [2], in this theory the entropy of an isolated instanton saturates the bounds (1.1) and (1.2) for a critical value of the 't Hooft coupling $\lambda_{t} \sim 1$. For a generic value of $\lambda_{t}$, the entropy scaling is similar to (2.17). More details can be found in [2] and shall not be repeated here. Instead, we wish to establish what is the significance of this fact from the point of view of the scattering amplitudes. Next, we wish to find out how the theory responds if we attempt to violate the bound by making $\lambda_{t}$ large.

First, we wish to show that the violations of the entropy bounds (1.1)-(1.2) by instanton (or any colored state) would result into violation of unitarity by the scattering amplitudes. We then argue that this is prevented by confinement. We shall try to support this statement by assuming the opposite and running into an inconsistency.

Indeed, assume that the theory never becomes confining. Yet, it is asymptotically free and therefore is consistent in UV. In such a theory there is no visible reason for why we cannot force an instanton of some size $R$ to violate the entropy bounds (1.1) and (1.2). This can always be achieved by making the 't Hooft coupling $\lambda_{t}$ arbitrarily large at that scale. 
However, the problem with this proposal is that simultaneously the unitarity would be violated by a $2 \rightarrow n$ scattering process with the momentum-transfer $q=\frac{1}{R}$. Consider a process in which the two initial gluons would scatter into $n$ final ones,

$$
A_{\beta}^{\gamma} A_{\gamma}^{\xi} \rightarrow A_{\beta}^{\alpha_{1}} A_{\alpha_{1}}^{\alpha_{2}} A_{\alpha_{2}}^{\alpha_{3}} \ldots A_{\alpha_{n-1}}^{\xi} .
$$

A typical 't Hooft diagram describing a process of this sort is given in figure 2. The color labels $\beta$ and $\xi$ are fixed by the initial gluons, whereas the color labels $\alpha_{j}(j=1, \ldots, n-1)$ take values from 1 to $N$.

Since, by assumption, the theory is not confining, the complete set of S-matrix asymptotic states can be represented by all possible $n$-gluon states with arbitrary color indexes,

$$
\left|A_{\beta_{1}}^{\alpha_{1}} A_{\beta_{2}}^{\alpha_{2}}, \ldots, A_{\beta_{n}}^{\alpha_{n}}\right\rangle .
$$

Of course, by symmetry, the final state vector $|t=\infty\rangle$, obtained as a result of Hamiltonian evolution, must transform under the same representation of the $\mathrm{SU}(N)$-group as the initial state $|t=-\infty\rangle$. That is, the state $|t=\infty\rangle$ must transform as a hermitian traceless $N \times N$ matrix with respect to the open color indexes $\xi$ and $\beta$. So, the true final state will be an appropriate superposition of all possible gluon states (4.5). In the current example this superposition will contain traces with respect to all indexes other than $\xi$ and $\beta$. Schematically,

$$
|t=\infty\rangle=\sum_{n} \sum_{\alpha_{1}, \ldots, \alpha_{n-1}} u_{n}\left|A_{\beta}^{\alpha_{1}} A_{\alpha_{1}}^{\alpha_{2}} A_{\alpha_{2}}^{\alpha_{3}} \ldots A_{\alpha_{n-1}}^{\xi}\right\rangle,
$$

where $u_{n}$ are some coefficients. The S-matrix elements will be determined by projecting this superposition on different individual states from the complete set (4.5). Correspondingly, in the rate of the process the squares of S-matrix elements are summed over all such states. In particular, for $2 \rightarrow n$ processes of the type (4.4) this amounts to,

$$
\sum_{\alpha_{1}, \ldots, \alpha_{n-1}}\left|\left\langle A_{\beta}^{\gamma} A_{\gamma}^{\xi}|\hat{S}| A_{\beta}^{\alpha_{1}} A_{\alpha_{1}}^{\alpha_{2}} A_{\alpha_{2}}^{\alpha_{3}} \ldots A_{\alpha_{n-1}}^{\xi}\right\rangle\right|^{2} .
$$

In order to avoid a potential confusion with the counting of the final states, we can softly Higgs the color group. We can easily achieve this by giving the tiny vacuum expectation values to a set of the "spectator" Higgs fields. Such a Higgsing of $\mathrm{SU}(N)$ symmetry generates a small mass gap and introduces the small mass splittings among the gluon fields. Since the theory is non-confining by assumption, this splitting affects neither the structure nor the magnitude of the amplitude. However, it removes all doubts whether the gluons of different colors must be counted as independent final states. We can then smoothly take the vacuum expectation values of the Higgs fields to zero and recover a gapless theory.

Note, in practice, the assumption that we are in an unconfining theory means that the scale $R=q^{-1}$, at which the entropy bound is violated, can be taken arbitrarily shorter than the length of the confinement, $L_{Q C D}$. For example, we can choose $L_{Q C D}$ to be of galactic size, whereas $q=R^{-1}$ to correspond to LHC energies. Obviously, in such a case a local LHC observer is not affected by the confinement. Such an observer would use the colored gluons (4.5), rather than the colorless composites such as glueballs, as the 
asymptotic states of the S-matrix. Can such an observer witness a violation of entropy by some field configuration at the scale $R$ ?

In order to argue against this, first assume that we are dealing with a fully resummed amplitude. Then, our previous discussion goes through and we skip the details. The summary is that the cross section of creating an each particular $n$-gluon state is exponentially suppressed. The enhancement is due to summation over micro-states corresponding to different color assignments of the final gluons, as expressed in (4.7). The resulting multiplicity factor is similar to (2.15). So, for $n=\frac{1}{\alpha}$ the cross section is given by (3.8). This cross-section saturates unitarity for $\lambda_{t} \sim 1$. This is strikingly close to a critical value for which, as observed in [2], the entropy of a single instanton of the same scale saturates both bounds, (1.1) and (1.2).

We now wish to see what happens if we try to violate these bounds by deforming the theory. We can achieve this by freezing $\lambda_{c}=1$ while increasing the 't Hooft coupling, $\lambda_{t} \rightarrow$ $\infty$. Of course, as already discussed, this would immediately result in a non-perturbative violation of unitarity by the process (4.4) since the cross section grows exponentially (4.3) with large $\lambda_{t}$. However, our point is that the confinement will set in before this can happen.

In other words, as already noted, by taking the theory not be confining, we have implicitly assumed that the scale of confinement $L_{Q C D}$ can be arbitrarily separated from the scale $R$ were the saturations of the entropy and unitarity bounds were taking place. Or equivalently, $L_{Q C D}$ can be arbitrarily larger than the saturon size $R$. What theory tells us is that this was a wrong assumption.

We shall now explain why. Indeed, the increase of $\lambda_{t}$ at a fixed scale $q$ represents a motion in the space of theories. This is because we are changing the relation between $\alpha(q)$ and $N$. However, alternatively, we can view the same deformation as a motion towards the IR-scale $q$ from some UV-scale $q^{\prime}>q$ within the same theory. Since we keep $\lambda_{c}=1$, this motion is accompanied by changing the number $n$ of gluon constituents in the final state. That is, within the same theory, we move from one process at the UV scale $q^{\prime}$ to a different process at the IR-scale $q$.

If gluons were to remain the valid degrees of freedom down to arbitrarily low energies, such a descend towards IR could be continued indefinitely. We would then sooner or later violate both entropy bounds (1.1) and (1.2). Correspondingly, the unitarity would also be violated. This would mean that the $\mathrm{SU}(N)$ gauge theory is inconsistent, despite being asymptotically free.

Somehow, the theory must prevent this from happening. In a theory with pure glue, the only visible mechanism that can prevent such an unlimited descend towards IR is confinement. That is, the theory must become confining before we manage to make $\lambda_{t}$ sufficiently large and violate both entropy bounds and unitarity. Thus, in a large- $N$ theory of pure glue the confinement appears to be a direct consequence of the bounds (1.1) and (1.2) and of the unitarity constraints imposed by them.

The above example with confinement is indicative in the following general sense. On one hand, the saturation of the entropy bound takes place for a critical value of the 't Hooft coupling that is typically order one. A further increase of $\lambda_{t}$ would make entropy larger than the value permitted by the bound (2.21). However, this is precisely the point 
at which the confinement sets in and prevents any further growth of $\lambda_{t}$. Of course, the confinement is a very special phenomenon belonging to a particular theory. However, the fact that the theory resists to an unlimited increase of the 't Hooft coupling beyond the critical point, appears to be genetic.

All the evidence we have, indicates that 't Hooft coupling is universally bounded from above by a critical value that is typically order one. When the system is pushed towards the strong 't Hooft coupling domain, it responds by a change of the regime which prevents a further growth of $\lambda_{t}$. In the above example of large- $N$ QCD with pure glue, the preventive mechanism is confinement, but the effect is very general.

We are observing that different systems respond differently to the increase of $\lambda_{t}$. In some non-confining systems, the further growth of $\lambda_{t}$ is simply impossible due to a dynamical energy balance. An example of this sort shall be considered in section 6 . In some other cases, for strong collective coupling, the configuration becomes unstable and chaotic. For example, such a behaviour has been confirmed by an exact numerical diagonalization of the Hamiltonian of a 1+1-dimensional system studied in [26].

\subsection{Baryons}

Notice, we encounter a similar resistance if we try to violate the entropy bound by quark bound-states. As observed in [1], a baryon of large- $N$ QCD [9] saturates the entropy bound when the number of quark flavors $N_{F}$ becomes of the same order as the number of colors $N$. Indeed, consider a baryon transforming as a symmetric tensor of rank $N$ under the flavor group $\mathrm{SU}\left(N_{F}\right)$. Its entropy is given by [1],

$$
S_{\mathrm{bar}} \simeq \frac{1}{\alpha} \ln \left(\left(1+\frac{\lambda_{c}}{\lambda_{F}}\right)^{\lambda_{F}}\left(1+\frac{\lambda_{F}}{\lambda_{c}}\right)^{\lambda_{c}}\right),
$$

where we have defined the analog of the 't Hooft coupling with respect to the global $\mathrm{SU}\left(N_{F}\right)$-flavor group, $\lambda_{F} \equiv \alpha N_{F}$. The baryon consists of $N$ quarks and has a size $R_{\mathrm{bar}} \sim$ $\Lambda_{Q C D}^{-1}$. Therefore, the collective coupling evaluated at the scale $q=R_{\mathrm{bar}}^{-1}$ is $\lambda_{c} \sim 1$. The above entropy then saturates the bound (1.2) for $\lambda_{F} \sim 1$. That is, the entropy reaches the allowed maximum for $N \sim N_{F}$.

Simultaneously, the area-law bound (1.1) as well as the Bekenstein bound (1.3) are also saturated. Indeed, remembering that the pion decay constant is $f_{\pi}=\sqrt{N} \Lambda_{Q C D}$ and the baryon mass is $M_{\mathrm{bar}}=N \Lambda_{Q C D}$, we can write,

$$
S_{\mathrm{bar}}=\frac{1}{\alpha}=\frac{1}{\alpha_{\pi}}=\left(R_{\mathrm{bar}} f_{\pi}\right)^{2}=M_{\mathrm{bar}} R_{\mathrm{bar}},
$$

where $\alpha_{\pi}=\frac{q^{2}}{f_{\pi}^{2}}$ is the pion coupling constant evaluated at the scale $q=R_{\mathrm{bar}}^{-1}=\Lambda_{Q C D}$.

It is natural that at the same time the $2 \rightarrow N$ pion scattering cross section saturates unitarity for the momentum-transfer set by the above scale $q$. This cross section is given by the expression analogous to (3.8) with $\lambda_{t}$ substituted by $\lambda_{F}$ and $\alpha$ by $\alpha_{\pi}$. This process can be interpreted as the production of a classical lump of the pion field. More precisely, the final state can be viewed as an overlapping pair of the pion solitons, i.e., skyrmions [10]. 
These solitons, as shown by Witten [11, 12], offer an effective description of the baryons at large $N$.

Now, we can try to violate the entropy bound by taking $\lambda_{F} \gg 1$. However, this is impossible because of the two reasons. First, this would make the theory asymptotically not free. Simultaneously, the above multi-pion scattering process would violate unitarity at the scale $q \ll R_{\text {bar }}^{-1}$. This would mean that the effective theory of pions breaks down at distances much larger than the would-be size of a baryon. So the latter object cannot even be described within such a theory. Of course, the two responses are related. Namely, the low energy theory of pions "senses" that something is going wrong in the UV and responds to it via violations of unitarity by multi-pion amplitudes. We thus observe that asymptotic freedom prevents the violation of the entropy bounds.

From the above point of view, the conformal window $[13,14]$ is of special interest. Since the coupling is at the fixed point, it appears that in such a regime the saturons with the fixed number of constituents $n=N$ and arbitrarily large sizes $R$ can exist. Correspondingly, their masses will assume values (3.10). As a result, the entropy of a saturon will be independent of its size and will be fixed at the bound (1.4). In this respect, such saturons would exhibit a scale-invariance.

\section{Scanning the cross section}

We now wish to scan the multi-particle cross section over different values of kinematic variables. For this, we need to parameterize $\sigma$ properly. First, we shall choose $n$ and $q$ as the scanning variables. Of course, in general, the number of active species $N$ can depend on the scale of momentum-transfer $q$. However, to start with, we assume $N$ to be independent of $q$. The scale-dependence of the 't Hooft coupling $\lambda_{t}(q)$ then is uniquely determined by the running of $\alpha(q)$. Thus, the cross section effectively depends on two parameters $(n, q)$, which can be traded for $\left(\lambda_{c}, \lambda_{t}\right)$ or $(E, q)$, and so on.

We shall perform the scanning in two different regimes. In the first case, we scan $n$ for fixed $q$. This is equivalent of scanning over $\lambda_{c}$ and $E$ while keeping $\lambda_{t}$ and $\alpha$ fixed. In the second case, we scan over $q$ (equivalently, over $\lambda_{t}$ and $E$ ) for the fixed values of $n$.

\subsection{Scanning $\lambda_{c}$}

We first freeze $\lambda_{t}, \alpha$ and the scale $q$ by the saturation condition (3.9) while allowing $n$ (equivalently $\lambda_{c}$ ) to vary. In this way, we scan over various processes in the same theory. These processes probe the same momentum transfer scale $q$ but differ by the occupation number $n$ in the final state. Obviously, they take place at different center of mass energies $E=n q$.

Now, when we move $\lambda_{c}$ away from its critical point, the resulting $n$-particle state saturates neither entropy bound nor unitarity. In order to see this, let us write the total $n$-particle cross section (3.8) for generic values of $\lambda_{c}$ and $\lambda_{t}$

$$
\sigma=\sigma_{2 \rightarrow n}\left(\left(1+\frac{\lambda_{t}}{\lambda_{c}}\right)^{\lambda_{c}}\left(1+\frac{\lambda_{c}}{\lambda_{t}}\right)^{\lambda_{t}}\right)^{\frac{1}{\alpha}}
$$


where

$$
\sigma_{2 \rightarrow n} \lesssim \begin{cases}\left(\lambda_{c}^{-1} \mathrm{e}\right)^{-\frac{\lambda_{c}}{\alpha}} & \text { for } \lambda_{c} \leqslant 1, \\ \mathrm{e}^{-\frac{\lambda_{c}}{\alpha}} & \text { for } \lambda_{c}>1 .\end{cases}
$$

As previously, using the power of large- $N$, the non-exponential prefactor is set equal to one. Of course, at the point of optimal truncation $\lambda_{c}=1$ the equation (5.1) reproduces (3.8). As already expressed by (2.18) and (3.9), at this critical point both entropy and unitarity are saturated for

$$
\lambda_{t}(q) \simeq 0.54
$$

Thus, if the running 't Hooft coupling reaches this critical value at some scale $q$, the theory gives rise to a saturon state in its spectrum. The mass and the radius of the saturon are given by (3.10) and (3.11) respectively.

The expression (5.1) shows that the cross section of $n$-particle state is peaked at $\lambda_{c}=1$ with the width $\sim \alpha$ and falls-off exponentially away from this point. In particular,

$$
\sigma \lesssim \begin{cases}\lambda_{t}^{\frac{\lambda_{c}}{\alpha}} & \text { for } \lambda_{c} \ll 1 \\ \left(\frac{\mathrm{e} \lambda_{c}}{\lambda_{t}}\right)^{\frac{\lambda_{t}}{\alpha}} \mathrm{e}^{-\frac{\lambda_{c}}{\alpha}} & \text { for } \lambda_{c} \gg 1\end{cases}
$$

This means that the saturon represents an exponentially narrow "resonance" of the width $\sim \alpha$ in the spectrum of all possible $n$-particle states of momentum $q$. As explained above, the momentum $q$ is defined by the criticality of the 't Hooft coupling (5.3).

Expressing $\lambda_{c}=\frac{E}{M}$ in terms of the center of mass energy $E=n q$ and the saturon mass $M=\frac{q}{\alpha}$, we can rewrite (5.4) as,

$$
\sigma \lesssim \begin{cases}\left(\lambda_{t}\right)^{\frac{E}{M \alpha}} & \text { for } E \ll M, \\ \left(\frac{\mathrm{e} E}{\lambda_{t} M}\right)^{\frac{\lambda_{t}}{\alpha}} \mathrm{e}^{-\frac{E}{M \alpha}} & \text { for } E \gg M .\end{cases}
$$

Now, remembering that $\lambda_{t} \simeq 0.54$, it is clear that away from the resonance energy $E=M$ the cross section is exponentially suppressed.

Thus, for producing a saturon in a 2-particle scattering experiment, the center of mass energy must be fine tuned to the mass of the saturon with an accuracy,

$$
\text { Saturation window : } \frac{\Delta E}{M} \sim \alpha \text {. }
$$

This illustrates the price that one needs to pay for producing a classical object with an unsuppressed cross-section in a renormalizable theory.

\subsection{Scanning $\lambda_{t}(q)$}

We now wish to scan the cross section over $q$ and $n$ while keeping $\lambda_{c}=1$. Then, the $q$-dependence enters the cross section (3.8) through the running 't Hooft coupling. Taking the derivative of (3.8) with respect to $q^{2}$, we get,

$$
\frac{d}{d q^{2}} \ln (\sigma) \simeq-N \ln \left(\left(1+\lambda_{t}\right) \mathrm{e}^{-1}\right)^{\frac{1}{\lambda_{t}}} \frac{d}{d q^{2}} \ln \left(\lambda_{t}\right)
$$


Around the saturation value (5.3) this expression simplifies to,

$$
\frac{d}{d q^{2}} \ln (\sigma) \simeq N \frac{d}{d q^{2}} \ln \left(\lambda_{t}\right)
$$

or equivalently,

$$
\frac{d \ln (\sigma)}{d \ln \left(\lambda_{t}\right)} \simeq N
$$

This result teaches us several things. First, around the saturation point the derivative of the cross section with respect to $\lambda_{t}$ scales as $N$. Thus, the scale-dependence of the cross section is extremely sensitive to the scale-dependence of $\lambda_{t}$.

Now, consider a theory that is asymptotically-free. In such a theory, $\lambda_{t}$ runs with $q^{2}$ logarithmically. Then, assuming we are not at the fixed point, we get,

$$
\frac{d \ln \left(\lambda_{t}\right)}{d \ln \left(q^{2}\right)} \sim \lambda_{t}
$$

Since, at the saturation point (5.3) the r.h.s. of the above equation is order one, the derivative is order one. Then, (5.8) tells us that the derivative of $\sigma$ around the same point is of order $N$. Thus, the cross section sharply diminishes as we move towards UV from the saturation point (5.3).

The motion towards infrared is more subtle. Obviously, any further increase of $\lambda_{t}$ is impossible without violating the entropy bound. Thus, we see the following two possibilities:

- Either the theory hits an infrared fixed point;

- Or it develops a mass-gap.

The latter can happen either due to confinement or a Higgs effect. In particular, as discussed above, in $\mathrm{SU}(N)$ gauge theory without matter, confinement appears to be the only mechanism that can prevent the violation of the entropy bound.

It is impressive how profound the quantum field theory is. It tells us that there is no "free-lunch" for producing a classical object in a two-particle scattering experiment at weakcoupling. This is true, despite the fact that the object saturates the cross section at the right energy $E=M$. The price is that the kinematic window of opportunity is very narrow.

It is certainly remarkable that a classical object can be produced with an unsuppressed cross-section in a renormalizable theory. However, it dominates the cross-section only for a particular "resonant" value of the center of mass energy. Away from it, the cross section falls off steeply. Fundamentally, the following trade-off takes place. The difficulty of producing a classical object in a quantum process manifests itself in an extremely precise choice of the center of mass energy in the scattering experiment.

Can saturons unitarize the cross section in a continuous range of energies? In renormalizable theories the difficulty is in maintaining the criticality relations such as $\lambda_{c} \simeq 1, \lambda_{t} \simeq 0.54$ over a continuous range of scales. In other words, the renormalizable theories do not possess saturons of arbitrary masses and sizes unless theory is at some non-trivial fixed point. 
Here comes a profound difference with non-renormalizable theories such as gravity. Gravity contains an almost continuous spectrum of saturons starting from the Planck mass and above. These saturons are black holes. This is the reason why gravity can selfunitarize by black holes at arbitrarily high center of mass energies above the Planck mass. It is interesting to confront how violations of the entropy bound and unitarity are avoided by the two theories. In $\mathrm{SU}(N)$ gauge theory this is achieved by confinement which generates a mass gap and forces the asymptotic states to be colorless. In contrast, in gravity the entropy violation is avoided by offering a black hole for arbitrarily high energy. In this way, the entropy is kept at the saturation point for arbitrarily high center of mass energy.

\section{A model of saturon as vacuum bubble}

We shall now come up with an explicit renormalizable theory that contains saturons. This theory allows us to take different parameter choices for which various entropy bounds are saturated by solitonic objects of different sizes and energy. We can then explicitly trace how the theory becomes inconsistent if Bekenstein bound (1.3) is obeyed without respecting the other two bounds (1.1) and (1.2). The conclusion is that a consistent theory must respect all three bounds and saturate all three of them simultaneously (1.4).

\subsection{The model}

Consider a theory of a scalar field $\phi$ that transforms as an adjoint representation of $\mathrm{SU}(N)$ symmetry. As usual, the latter can be written as $N \times N$ traceless hermitian matrix $\phi_{\alpha}^{\beta}$, where $\alpha, \beta=1,2, \ldots, N$. In order not to blur the effect by the confinement, we shall keep the $\mathrm{SU}(N)$-symmetry global. The Lagrangian of the theory is,

$$
L=\frac{1}{2} \operatorname{Tr}\left(\partial_{\mu} \phi \partial^{\mu} \phi\right)-V(\phi),
$$

where the scalar potential has the form,

$$
V(\phi)=\frac{\alpha}{2} \operatorname{Tr}\left(f \phi-\left(\phi^{2}-\frac{I}{N} \operatorname{Tr} \phi^{2}\right)\right)^{2} .
$$

Here, $I$ is the unit $N \times N$ matrix. The vacuum equations,

$$
f \phi_{\alpha}^{\beta}-\left(\phi^{2}\right)_{\alpha}^{\beta}+\frac{\delta_{\alpha}^{\beta}}{N} \operatorname{Tr} \phi^{2}=0
$$

have many degenerate solutions. They correspond to spontaneous breaking of $\mathrm{SU}(N)$ symmetry down to $\mathrm{SU}(N-K) \times \mathrm{SU}(K) \times \mathrm{U}(1)$ subgroups for values of $0<K<N$. In addition there exists an unbroken symmetry vacuum with $\phi_{\alpha}^{\beta}=0$.

All the above vacua are equally good for our purposes. So, for definiteness, we shall focus on the unbroken-symmetry vacuum $\phi=0$ and the one with $K=1$. In the latter vacuum only the following component

$$
\phi_{\alpha}^{\beta}=\phi(x) \operatorname{diag}((N-1),-1, \ldots,-1) \frac{1}{\sqrt{N(N-1)}},
$$


has a non-zero expectation value. Up to irrelevant $1 / N$-corrections, this expectation value is equal to

$$
\langle\phi\rangle=f .
$$

Due to spontaneous breaking of global $\mathrm{SU}(N)$ symmetry down to $\mathrm{SU}(N-1) \times \mathrm{U}(1)$, this vacuum houses massless Goldstone species. Their number is,

$$
N_{\text {Gold }}=2(N-1),
$$

and their decay constants are given by $f$. As usual, the coupling "constant" of these Goldstones, which we denote by $\alpha_{\text {Gold }}$, exhibits the following dependence on the scale of momentum-transfer $q$,

$$
\alpha_{\text {Gold }}=\frac{q^{2}}{f^{2}} .
$$

Correspondingly, we define the 't Hooft coupling for Goldstones,

$$
\lambda_{\text {Gold }} \equiv \alpha_{\text {Gold }} N_{\text {Gold }} \simeq 2 N \frac{q^{2}}{f^{2}} .
$$

Since the vacuum (6.4) is exactly degenerate with the one with unbroken symmetry, there exist domain walls that separate the two. The solution for a planar infinite wall has the form,

$$
\phi(x)=\frac{f}{2}\left(1 \pm \tanh \left(\frac{x m}{2}\right)\right),
$$

where $x$ is a coordinate that is perpendicular to the wall. The tension (energy per unit surface area) of the wall is given by,

$$
\mu=\frac{1}{6} \frac{m^{3}}{\alpha},
$$

and the thickness of the wall is,

$$
R \sim \frac{1}{m} .
$$

Approximately, the same expressions apply to a closed bubble when its radius $r$ is much larger than the wall thickness, $r \gg R \sim m^{-1}$. This regime is usually referred to as the thin wall approximation.

In the regime of our interest, in which $\alpha$ is very small, the bubbles are long-lived. That is, they oscillate for a sufficiently long time before decaying into particles. The qualitative way for understanding this stability is different for large and for small bubbles. For large bubbles $\left(r \gg m^{-1}\right)$ the oscillation frequency is $\sim 1 / r$. This is much less that the mass of a free quantum. Consequently, the production rate is suppressed. The decay rate for the small bubbles, $r \sim m^{-1}$, will be derived later. However, a qualitative reason for their long life-time is that the decay goes through the quantum re-scattering of constituents which is suppressed due to weak coupling.

Notice, if we restrict the adjoint field to its component (6.4), the potential (6.2) becomes

$$
V(\phi)=\frac{\alpha}{2}\left(f \phi-\phi^{2}\right)^{2}+\mathcal{O}\left(N^{-2}\right) .
$$


We now wish to derive the restrictions imposed on the theory by the three entropy bounds, (1.3), (1.2) and (1.1) and by unitarity. We start by choosing the trivial vacuum $\phi=0$ as our asymptotic S-matrix vacuum. In this vacuum all particles have a mass $m=\sqrt{\alpha} f$. Next, consider a vacuum bubble inside of which $\phi=f$.

The crucial fact is that inside the bubble the $\mathrm{SU}(N)$-symmetry is spontaneously broken down to $\mathrm{SU}(N-1) \times \mathrm{U}(1)$ subgroup. This breaking results into $\sim 2 N$ gapless Goldstone modes localized within the bubble world-volume. These Goldstone modes create an exponentially large number of the bubble micro-states. Using the method of [1], we can estimate this number in the following way. The degeneracy of the bubble interior is controlled by the degeneracy of the vacuum manifold in the broken phase. This vacuum manifold is obtained by the action of $\mathrm{SU}(N) / \mathrm{SU}(N-1) \times \mathrm{U}(1)$ transformations on the expectation value (6.4). The effective quantum Hamiltonian that describes the corresponding degeneracy of the bubble is:

$$
\hat{H}=X\left(\sum_{j} \hat{a}_{j}^{\dagger} \hat{a}_{j}-s(r)\right),
$$

where $\hat{a}_{j \text {-s }}$ are quantized zero modes that classically parameterize the bubble moduli space. Their number is of order $2 N$. The quantity $s(r)$ is the time-averaged space integral of $\phi^{2}(x)$. For large (and slow) bubbles, $r \gg m$, for which the thin wall approximation works, it is given by the bubble volume times $m f^{2}$,

$$
s(r) \simeq \frac{4 \pi}{3} r^{3} m f^{2}=\frac{4 \pi}{3} \frac{(r m)^{3}}{\alpha},
$$

whereas for the smallest bubbles, $r \sim m^{-1}$, we have $s \sim \frac{1}{\alpha}$.

Now, the degeneracy of (6.13) is given by the binomial factor which is of order

$$
n_{\mathrm{st}}(r) \sim\left(1+\frac{2 N}{s(r)}\right)^{s(r)}\left(1+\frac{s(r)}{2 N}\right)^{2 N}
$$

This degeneracy endows the bubble with the corresponding micro-state entropy $S_{\mathrm{bub}}(r)=$ $\ln \left(n_{\mathrm{st}}(r)\right)$. Next, for convenience, we introduce a notation,

$$
\lambda(r) \equiv \frac{2 N}{s(r)}=\frac{2 \lambda_{t}}{\alpha s(r)},
$$

where the 't Hooft coupling $\lambda_{t}$ is defined as before, (2.3). In this notations, we can write the entropy of a bubble of radius $r$ as

$$
S_{\mathrm{bub}}(r)=s(r) \ln \left((1+\lambda(r))\left(1+\frac{1}{\lambda(r)}\right)^{\lambda(r)}\right) .
$$

We shall now investigate the response of the theory when the above entropy saturates the three bounds (1.3), (1.1) and (1.2) for the bubbles of various sizes. 


\subsubsection{Small bubbles as saturons}

We consider the smallest bubbles first, $r \sim R=m^{-1}$. The energy and the surface area of such a bubble are given by $E_{\mathrm{bub}} \sim \frac{1}{R \alpha}$ and Area $\sim R^{2} \sim m^{-2}$ respectively. Correspondingly, for such bubbles we have,

$$
E_{\mathrm{bub}} R \sim \frac{1}{\alpha} \sim \frac{1}{\alpha_{\text {Gold }}} \sim(R f)^{2} .
$$

Thus, all three bounds: the Bekenstein bound (1.3), the inverse-coupling bound (1.2) and the area-law bound (1.1) are satisfied simultaneously. Moreover, the inverse-coupling bound is satisfied for both couplings: for the coupling of massless Goldstones, $\alpha_{\text {Gold }}$, as well as, for the coupling of massive $\phi$-quanta, $\alpha$. The reason is that the range of the interactions mediated by both fields is large enough to cover the size of the smallest bubble $r \sim m^{-1}$. Correspondingly the bound (1.2) must be satisfied with respect to both couplings, and it is. To put is shortly, we see that for smallest bubbles the relation (1.4) holds.

From the definition (6.16) and the expression (6.17) it is easy to see that the above saturation takes place when the both 't Hooft couplings are order one,

$$
\text { Saturation point: } \lambda_{t} \sim \lambda_{\text {Gold }} \sim 1 \text {. }
$$

Using our previous knowledge, it is easy to see how the above saturation of the entropy bound is mapped on the saturation of unitarity. Namely, in respective S-matrix vacua the processes $2 \rightarrow n$ saturate unitarity at momentum transfer $q=m$. Of course, in both vacua, the saturation takes place at the points of optimal truncation.

A typical process of this sort is given by figure 2. Here the double lines must be understood as the adjoint $\phi$-field in 't Hooft's notations. For such processes, our previous analysis is directly applicable. As we already discussed in details, the cross section of this process is given by (3.8). Obviously, in this expression we must insert the couplings that are relevant for a given process. For example, for $2 \rightarrow n$ Goldstone scattering process in $\mathrm{SU}(N-1) \times \mathrm{U}(1)$ vacuum, at the point of optimal truncation $n=\alpha_{\text {Gold }}^{-1}$ the cross section will take the form,

$$
\sigma_{\text {Gold }}=\left(\mathrm{e}^{-1}\left(1+\lambda_{\text {Gold }}\right)\left(1+\frac{1}{\lambda_{\text {Gold }}}\right)^{\lambda_{\text {Gold }}}\right)^{\frac{1}{\alpha_{\text {Gold }}}} .
$$

As we already discussed several times, the above cross section is saturated for $\lambda_{\text {Gold }}$ order one.

It is not surprising that this matches a regime in which the vacuum bubble saturates the entropy bound (1.4). Indeed, from the point of view of an S-matrix vacuum with unbroken symmetry, the smallest bubbles are well described as self-sustained states of weakly interacting quanta of occupation number $n=\frac{1}{\alpha}$. Correspondingly, the $n$-particle process that saturates unitarity can be viewed as describing the formation of such a bubble in a two-particle scattering process.

As we have discussed previously, the processes with the higher number of the final quanta are exponentially suppressed. The reason was that, once we saturate the entropy 
bound by a state $\lambda_{c}=1$, all the states $\lambda_{c} \gg 1$ are well below the bound. As a result, their entropy factors are too weak for winning over the exponential suppression of the amplitudes. This is clearly illustrated by the equations (5.4) and (5.5).

The above insufficiency of the entropy enhancement for the states with $\lambda_{c} \gg 1$ is also matched by the entropy count of the larger bubbles $r \gg m^{-1}$. In order to see this, first check the entropies of such bubbles. From (6.16) and (6.14) it is clear that for large bubbles we have

$$
\lambda(r) \simeq \frac{3 \lambda_{t}}{2 \pi(r m)^{3}}
$$

Recall that the 't Hooft coupling was already set to its critical value $\lambda_{t} \sim 1$ by the requirement of entropy saturation by the smallest bubbles. Since, $\lambda_{t}$ is a parameter of theory, it is the same for the bubbles of all sizes. Then, from (6.17) and (6.21) it is clear that for the large size bubbles the entropy scales as,

$$
\left.S_{\mathrm{bub}}(r)\right|_{r \gg m^{-1}} \simeq \frac{2 \lambda_{t}}{\alpha} \ln \left(\frac{2 \pi \mathrm{e}(r m)^{3}}{3 \lambda_{t}}\right)
$$

It is not difficult to see that the above entropy is well below of all three bounds (1.3), (1.1) and (1.2).

Indeed, the maximal entropy permitted by the Bekenstein bound (1.3) for a large bubble has the form,

$$
S_{\mathrm{Bek}}(r)=2 \pi E_{\mathrm{bub}} r \simeq \frac{4 \pi^{2}}{3} \frac{(r m)^{3}}{\alpha} .
$$

Obviously, this is much larger than (6.22).

Next, check the inverse coupling bound (1.2). Since the bubble is much larger than $m^{-1}$, the only interaction that has a relevant range is the Goldstone exchange. Remembering that the Goldstone coupling (6.7) evaluated at $q=\frac{1}{r}$ is $\alpha_{\text {Gold }}=(f r)^{-2}$, the corresponding entropy bound is

$$
S_{\text {Gold }}(r)=\frac{1}{\alpha_{\text {Gold }}}=(f r)^{2}=\frac{(m r)^{2}}{\alpha} .
$$

Since the Goldstone coupling constant is equal to the inverse area of the bubble measured in units of $f$, the last expression also accounts for the area-law entropy bound (1.1). As we can see, both are way larger than the actual entropy of the large bubble (6.22).

To summarize, we see that when the smallest bubble saturates the entropy bound, it saturates all three bounds simultaneously, (1.4). At the same time, the larger bubbles are below the bound. Correspondingly, their entropies cannot compete against the exponential suppressions of the respective amplitudes.

\subsubsection{Suppression of large bubbles}

We wish to explicitly demonstrate the insufficiency of the entropy enhancement of the cross sections for creation of large bubbles in a two-particle scattering process in the regime in which the smallest bubbles saturate the entropy bound. We can achieve this by applying our results to the analysis of [18]. In this work a process or bubble-creation in thin wall 
approximation was studied in a theory of a single real scalar field $\phi$ with two degenerate vacua. Naturally, since such bubbles carry zero entropy, no entropy enhancement was discussed there. Notice, our theory would reduce to such a model if we would reduce it to a single component (6.4) of the adjoint field. The resulting theory of course contains vacuum bubbles similar to ones we have studied. However, they carry zero entropy due to the absence of the Goldstone phenomenon in the bubble interior. Therefore, the bubble production rates in [18] and in the present model (6.1) will differ by the entropy factor.

In [18] the creation of a vacuum bubble of energy $E=n m$ from a single virtual quantum was studied as the first stage of a two-stage process. The second stage amounts to a decay of the bubble into $n$ near-mass-threshold particles. We shall focus on the first part of the process. In our notations, the matrix element of [18] describing the bubble-formation has the form,

$$
\left|A_{1 \rightarrow B}\right|^{2} \sim \mathrm{e}^{-c n \sqrt{\lambda_{c}}}
$$

where $c>0$.

Now, the novelty in our case is that the rate must be summed over a large number of the bubble micro-states. This amounts to multiplying (6.25) by the degeneracy factor $\mathrm{e}^{S_{\mathrm{bub}}}$. For the large bubbles the entropy is given by (6.22). Noticing that $\lambda_{c}=\frac{E_{\mathrm{bub}}}{m} \alpha=\frac{2 \pi}{3}(m r)^{2}$, the large bubble entropy can be written as $S_{\text {bub }}(r) \simeq \frac{3 \lambda_{t}}{\alpha} \ln \left(\lambda_{c}\right)$. The rate of the bubble production is then given by,

$$
\Gamma \sim\left|A_{1 \rightarrow B}\right|^{2} \mathrm{e}^{S_{\mathrm{bub}}} \sim \mathrm{e}^{-n \sqrt{\lambda_{c}}\left(c-3 \lambda_{t} \frac{\ln \left(\lambda_{c}\right)}{\lambda_{c} \sqrt{\lambda_{c}}}\right)} .
$$

Now, remembering that in the above expression $\lambda_{t} \sim 1$ and $\lambda_{c} \gg 1$, it is clear that the entropy enhancement factor is negligible as compared to the suppression. So, the production rate of the large bubbles continues to be exponentially suppressed despite the entropy enhancement.

Of course, the situation is very different for the smallest bubbles, $r \sim m^{-1}$, that saturate the entropy bound (1.4). Because of this, they also saturate unitarity in the scattering process and are produced by an unsuppressed rate. This is also indicated by saturation of unitarity by the corresponding $n$-particle scattering process.

Unfortunately, the analysis of [18] is not applicable for small bubbles. Such bubbles correspond to $\lambda_{c} \sim 1$, which is outside of the validity domain of [18]. However, extrapolating (6.26) towards $\lambda_{c} \sim 1$, clearly shows the tendency: the entropy factor starts to compensate the suppression term. Of course, this is fully consistent with our results of saturating the $n$-particle cross section at the optimal truncation point. This is natural since the smallest bubbles are well-described as $n$-particle states. Correspondingly, the two pictures - producing a bubble or an $n$-particle state - must match.

\subsubsection{Superiority of area-law and inverse-coupling bounds}

We now wish to show that saturating the Bekenstein bound (1.3) while disrespecting the bounds (1.2) and (1.1) leads to an inconsistency of the theory. This indicates that in general the latter bounds are more stringent than the former one. 
In the present model this happens when a large bubble of certain radius $r_{*} \gg m^{-1}$ saturates the Bekenstein entropy bound (1.3). As we shall see, such a saturation violates the other two bounds (1.1) and (1.2) and this triggers the violation of unitarity by the scattering amplitudes.

The saturation value of $\lambda\left(r_{*}\right)$ can be found by equating (6.17) to the corresponding Bekenstein entropy (6.23). Using the expression (6.14), this saturation condition can be written in the following form,

$$
\left(1+\lambda\left(r_{*}\right)\right)\left(1+\frac{1}{\lambda\left(r_{*}\right)}\right)^{\lambda\left(r_{*}\right)} \simeq \mathrm{e}^{\pi},
$$

which is satisfied for $\lambda\left(r_{*}\right) \simeq 8$.

At first glance this saturation looks rather innocent. However, meanwhile the bounds (1.1) and (1.2) are violated both by the Goldstone coupling $\alpha_{\text {Gold }}$ and the decay constant $f$. This is immediately clear by comparing the maximal entropy (6.24) permitted by the area (1.1) and the inverse-coupling (1.2) bounds to the Bekenstein entropy of the same bubble (6.23). We have,

$$
\frac{S_{\mathrm{Bek}}\left(r_{*}\right)}{S_{\mathrm{Gold}}\left(r_{*}\right)}=\frac{4 \pi^{2}}{3}\left(m r_{*}\right) \gg 1 .
$$

The violation of the inverse-coupling (1.2) and the area-law (1.1) bounds, leads to the following disaster.

First notice, that the corresponding value of the Goldstone 't Hooft coupling is enormously large,

$$
\lambda_{\text {Gold }} \simeq \frac{32 \pi}{3}\left(r_{*} m\right) \gg 1 .
$$

This is a very serious problem for the theory. With such a strong 't Hooft coupling, the $2 \rightarrow n$ Goldstone scattering process in $\mathrm{SU}(N-1) \times \mathrm{U}(1)$ vacuum, violates unitarity at the point of optimal truncation $n=\alpha_{\text {Gold }}^{-1}$. Indeed, the cross section (6.20) for large Goldstone 't Hooft coupling given by (6.29) scales as,

$$
\sigma \simeq\left(\lambda_{\text {Gold }}\right)^{\frac{1}{\alpha_{\text {Gold }}}}=\left(\frac{32 \pi}{3}\left(r_{*} m\right)\right)^{\frac{\left(r_{*} m\right)^{2}}{\alpha}} .
$$

Since in this expression $\left(r_{*} m\right) \gg 1$, the above cross section violates unitarity beyond any repair.

Now, the important thing is that the above violation takes place for the momentumtransfer $q \sim r_{*}^{-1}$. The physical meaning of this fact is that the actual UV-cutoff of the theory in the Goldstone vacuum is much less than the scale $r_{*}^{-1}$,

$$
\Lambda_{U V} \ll \frac{1}{r_{*}} .
$$

This means that the bubble of size $r_{*}$ cannot be described within the validity of the theory. This is despite of the fact that the bubble respects the standard Bekenstein bound (1.3). It is the violation of the other two bounds (1.1) and (1.2) that makes the theory inconsistent. 
We thus arrive to the following conclusion:

A violation of the inverse-coupling (1.2) and the area-law (1.1) entropy bounds makes the theory inconsistent even if the standard Bekenstein bound (1.3) is satisfied.

Thus, the inverse-coupling (1.2) and the area-law (1.1) entropy bounds are not equivalent to Bekentein bound (1.3) and in fact are more stringent. On the other hand, saturation of (1.1), (1.2) also implies saturation of (1.3). Thus, in a consistent theory all three bounds must be respected and saturated simultaneously.

\section{$7 \quad$ Black holes as saturons}

Obviously, there are striking parallels exhibited by saturons in renormalizable theories on one hand and black holes in gravity on the other. These parallels appear to be so vast and so precise that they must indicate about the universality of physics-laws that govern the saturation point (1.4). This universality goes way beyond the particularities of the underlying theory, whether it is gravity, a gauge theory or something entirely different. What we are learning is that physics is controlled by a fundamental connection between entropy and unitarity expressed by the bound (1.4).

In this section we shall make these parallels more transparent by organizing them in form of an explicit "checklist" of similarities between renormalizable saturons and black holes. In order to make the extend of the connection brisk, we shall choose for the role of non-gravitational saturons the vacuum bubbles of the theory given by (6.1). We remind the reader that the latter is a renormalizable quantum field theory of a self-interacting scalar field $\phi$ in the adjoint representation of $\mathrm{SU}(N)$ symmetry. Since this symmetry is not even gauged, it is hard to imagine an example that is more distant from gravity. Nevertheless, as we shall see, the saturons in this theory share all their key properties with black holes. We shall now discuss these properties one by one.

\subsection{Similarities in entropy}

As already discussed in details, saturons in the theory (6.1) represent vacuum bubbles. An exterior of the bubble is an unbroken symmetry vacuum which we choose as asymptotic S-matrix vacuum for our observer Alice. In the interior of the bubble the $\mathrm{SU}(N)$ symmetry is spontaneously broken down to a maximal subgroup which we chose as $\mathrm{SU}(N-1) \times \mathrm{U}(1)$. This breaking results into $\sim N$ Goldstone bosons localized in the bubble world volume. They endow the bubble with the entropy given by (2.17). As already explained, the alternative way to think about bubble entropy is in terms of group representations. Because the bubble is not elementary but rather is a state with high occupation number, it transforms as a large representation of the $\mathrm{SU}(N)$ group. The entropy is set by the log of the dimensionality of this representation. As we have seen, only the smallest bubbles, of size $r \sim R=m^{-1}$, can saturate the entropy bound consistently. At the saturation point they saturate all three bounds (1.3), (1.1) and (1.2) simultaneously. Therefore, they satisfy the relation (1.4). 
Now, we wish to note that (1.4) is exactly the relation satisfied by the Bekenstein entropy of a black hole [5]. Of course, the fact that black hole entropy saturates the ordinary Bekenstein bound (1.3) and also exhibits the area law, is well-known. What is much less appreciated is that the black hole entropy also saturates the inverse-coupling bound (1.2). The latter observation was originally made in $[6,7]$ which we shall now explain.

For this, first note that the graviton coupling at the scale of momentum-transfer $q$ is given by,

$$
\alpha_{\mathrm{gr}}(q)=\frac{q^{2}}{M_{P}^{2}} .
$$

However, this is nothing but an inverse of the Bekenstein entropy of a black hole of radius $R=q^{-1}$ ! Thus, the entropy of a black hole of mass $M$ and radius $R=\frac{M}{M_{P}^{2}}$ obeys the following relation,

$$
S_{\mathrm{BH}}=M R=\frac{1}{\alpha_{\mathrm{gr}}(q)}=\frac{\text { Area }}{M_{P}^{-2}} .
$$

This is exactly the relation (1.4) with $f=M_{P}$ and $q=1 / R$. As already explained in the introduction, the relation is obvious since $M_{P}$ represents the graviton decay constant. Also, a black hole breaks translation symmetry spontaneously and the Goldstone mode of this breaking is of course the graviton excitation.

\subsection{Decay and life-time}

Until now, the best understood computation about the decay of a black hole, is the famous original one by Hawking [30]. This computation is exact in the following semi-classical limit,

$$
M \rightarrow \infty, \quad M_{P} \rightarrow \infty, \quad R=\text { finite } .
$$

Of course, simultaneously the Planck constant $\hbar=1$ is kept finite. Notice, in the above limit, also the black hole entropy $S_{\mathrm{BH}}$ becomes infinite, as it is clear from (7.2).

Now, in the limit (7.3) the geometry of a black hole experiences no back-reaction from the emitted quanta. That is, a black hole becomes a rigid reservoir of infinite energy and information capacity. The Hawking's computation shows that in this limit black hole emits in thermal spectrum with temperature $T \sim \frac{1}{R}$. That is, on average, a black hole emits a quantum of energy $\sim \frac{1}{R}$ per time $\sim R$. The emission of more energetic quanta is exponentially suppressed, whereas the less energetic ones are suppressed by the phase-space.

Of course, in the limit (7.3) the black hole mass is infinite and so is the life-time. However, if we extrapolate Hawking's result for finite $M$, we can estimate that the black hole shall lose of order half of its mass approximately after the time,

$$
t_{\mathrm{BH}} \sim R\left(R M_{P}\right)^{2} \sim R S_{\mathrm{BH}} .
$$

The last part of the equation relates this time-scale with the black hole entropy. This is indicative, since the number of the emitted quanta of energy $\sim 1 / R$ is equal to the black hole entropy.

Now, strictly speaking, it is unjustified to extrapolate the results of Hawking's semiclassical computation beyond the above time-scale. The reason, without entering into much 
guess-work about the microscopic quantum gravity, is simple [32]. The back-reaction, that the black hole experiences with each emission, is of order $\sim \frac{1}{S_{\mathrm{BH}}}$. So, the cumulative effect after the time (7.4) is large and must be taken into account. This cannot be done without working in an explicit microscopic theory in which we shall not enter. We shall therefore limit the study of the connection between saturons and black holes by the time-scale (7.4).

We now wish to show that the quantum decay of a saturon bubble exhibits a very similar behaviour. Let us first note that the long life-time of large bubbles was concluded in the earlier studies both by numerics [27-29] as well as by analytic arguments [18]. The latter argument relies on a very narrow level-spacing of quantized bubbles. Due to this, the emission of particles requires transitions between distant levels which is suppressed by the wave-function overlap. In the present case there will be an additional suppression factor due to the memory burden effect $[35,36]$. This effects is connected with the high entropy of the bubble which stabilizes it against the spread-out. Assuming that classically the bubble is long lived, we focus our interest on the smallest ones that saturate the entropy bounds and satisfy (1.4).

Now, for a saturon bubble of the theory (6.1), the analog of Hawking's semi-classical limit (7.3) is

$$
M \rightarrow \infty, \quad f \rightarrow \infty, \quad R=\text { finite }
$$

or equivalently,

$$
S_{\text {bubble }}=\frac{1}{\alpha} \rightarrow \infty, \quad \lambda_{c}=1, R=\text { finite } .
$$

In this limit, the decay rate of the saturon can be estimated in the following way.

The saturon bubble represents a loose bound-state of bosons of mass $m$. Because of the binding potential their energies are of course below the threshold of free quanta. However, the particles can be emitted because of quantum depletion due to re-scattering. The rate can be easily estimated and is given by (see, $[6,7]$ for a very similar estimate of the depletion of a saturated state),

$$
\Gamma_{\text {emission }} \sim R^{-1} \alpha^{2} n^{2} \sim R^{-1}
$$

Thus, just like a black hole, the saturon emits on average one quantum of energy $\sim \frac{1}{R}$ per time $\sim R$. The emission of more energetic quanta is exponentially suppressed because this requires a re-scattering of larger number of constituents. At the same time, the low energy ones are suppressed by the phase space. Of course, since theory has a mass gap, nothing can be emitted below the energy $\sim m$.

To summarize, an asymptotic observer, Alice, would see a saturon bubble as an object that emits in approximately-thermal spectrum. This is true despite the fact that the $n$ particle state of saturon is not really thermal. What creates the effect of thermality is the softness of the constituent quanta and the fact that the state is at the critical point $\lambda_{c}=1$. Now, extrapolating this result to finite $n$, the resulting half-life time of saturon bubble is,

$$
t_{\text {bub }} \sim R(R f)^{2} \sim R S_{\text {bub }}
$$

Without much commenting, the striking analogy with all the aspect of black hole evaporation and in particular with its half-life (7.4) is obvious. 


\subsection{Infomation horizon and time-scales}

One of the characteristic properties of semi-classical black holes (7.3) is the existence of the horizon. This makes an information about the black hole interior inaccessible for an outside observer, such as Alice. It is widely believed, although remains a subject of active controversy, that for a black hole of finite mass the information is no longer hidden and finally comes out. We shall not question this point of view since within a consistent particle physics framework with unitary S-matrix, no other outcome is imaginable for us. The question therefore is not whether the information is accessible but rather how long is the required time-scale for decoding it. Of course, it is reasonable to assume that the minimal time-scale required for a start of the information read-out, is the half-life of a black hole. This view is supported by general arguments by Page [31]. We shall therefore adopt the equation (7.4) as the lower bound on such a time-scale.

We shall now see that all the above properties are matched by saturons of renormalizable theory (6.1). Of course, the advantage is that in case of a saturon bubble we can understand the microscopic origin of such properties very transparently. Let us first notice that, just like a black hole, a saturon bubble creates an information horizon that makes the knowledge about its micro-state inaccessible for Alice. Indeed, the quantum information is encoded in saturon micro-states. These micro-states are labelled by the excitations of the gapless Goldstone modes that are confined to the interior of the saturon. Their number is $\sim N$ as it is also indicated by the entropy of the saturon.

Now, for reading out this information Alice faces the following dilemma:

- Alice can wait for Saturon evaporation and examine its decay products very carefully;

- Alternatively, Alice can scatter an external probe particle at the saturon and study the outcome.

A slight technical problem with pursuing both methods simultaneously is that scattering will in general alter the internal state of saturon. So, it is cleaner to follow one protocol.

It is easy to see that the minimal time-scale required by both efforts is given by (7.8). Indeed, in order to examine the decay products carefully, Alice has to setup an interaction that distinguishes among the different states within the same $\mathrm{SU}(N)$-multiplet. This is similar to measuring a spin polarization of a particle in a theory with a rotationally-invariant Hamiltonian. Despite the fact that Hamiltonian commutes with the spin operator, the particle spin projection can still be measured. This is not an issue. The problem in case of a saturon bubble is that the information is stored among the states of enormous number of Goldstone modes. So, each emitted quantum carries only a tiny fraction of this information.

The rate by which an emitted quantum interacts with Alice's device is,

$$
\Gamma_{\text {Alice }} \sim \frac{1}{R} \alpha^{2} \mathcal{N}_{\text {Alice }}
$$

where $\mathcal{N}_{\text {Alice }}$ is the measure of the capacity of the device which is under Alice's control. Alice can maximize this capacity, for example, by preparing a huge reservoirs of probe 
particles. However, even if Alice manages to identify the state of a given emitted quantum, the latter only carries an exponentially small part of the information about the state of the entire saturon. So, Alice needs to gather at least of order $\sim n$ emitted quanta before she can start decoding information at a reasonable rate. This requires a minimal waiting time given by (7.8), in exact analogy with a black hole.

Now, the second option for Alice is to scatter a soft probe particle through the interior of the saturon and study the scattering products. The hope is that the probe particle shall interact with Goldstone bosons that are confined within the interior and bring out the information about their state. Notice, the probe particle must be optimally soft: on one hand, it should not create too much level-splitting among the states of the gapless Goldstones and, on the other hand, the interaction rate must not be too low. The latter rate is suppressed by the decay constant of Goldstone bosons $f$.

At the end, the rate of scattering between an optimally-soft probe and the saturon Goldstone field is,

$$
\Gamma_{\text {Gold }} \sim \frac{1}{R^{3} f^{2}}
$$

The corresponding time-scale is nothing but a half-life of the saturon bubble (7.8). Again, we observe that similarity with the black hole case is complete. In particular, in the limit (7.5) the information becomes inaccessible. This is exactly analogous to what happens with black hole information in the limit (7.3). Of course, both limits are fully consistent with unitarity since the respective objects become infinitely massive and their life-times become eternal.

\subsection{Scattering amplitudes}

As the last step for completing the list of similarities between non-gravitational saturons and black holes, we discuss relation with scattering amplitudes. As we have shown, the saturation of entropy bound (1.4) by a bubble (or any other soliton) is in one to one correspondence with the sturation of unitarity by the respective $2 \rightarrow n$ scattering process. We wish to point out that this connections carries over into black holes. The idea that a black hole can be produced in a collision of few particles of center of mass energy $E \gg M_{P}$ is not new and goes back to [37-41] and many subsequent papers. However, only relatively recently [42-44], this process has been connected to $2 \rightarrow n$ graviton scattering amplitudes. The actual detailed computation of the amplitude was performed in [43] and [44]. The study was motivated by the microscopic picture of $[6,7]$ in which a black hole is described as $n$-graviton state at the point of saturation $\lambda_{c}=1$. However, in the present discussion we would prefer not to have any microscopic bias.

So, we put ourselves in the position of Alice, who is making no assumption about the microscopic theory of a black hole. Alice is simply observing a process of black hole formation in a collision of two quanta of center of mass energy $E \gg M_{P}$ and its subsequent evaporation into $n$ soft ones. It is obvious that the process that Alice identifies as a relevant S-matrix process is $2 \rightarrow n$. 
This is exactly the computation performed in [43]. The resulting cross section of producing a particular $n$-graviton state is

$$
\sigma_{2 \rightarrow n}=n !\left(\alpha_{\mathrm{gr}}\right)^{n} .
$$

The crucial point is that the above expression reduces to,

$$
\sigma_{2 \rightarrow n}=\mathrm{e}^{-\frac{1}{\alpha_{\mathrm{gr}}}}=\mathrm{e}^{-S_{\mathrm{BH}}},
$$

exactly when the softness of outgoing gravitons matches the Hawking quanta $q=\frac{1}{R}$. Now, strictly speaking, we have no moral obligation to interpret these $n$-graviton states as the black hole micro-states. However, intuitively the connection is clear. So, we can interpret them as "relatives". This relation carries the same meaning as the relation between the saturon vacuum bubble in theory (6.1) and the $n$-particle state into which it decays. It is then clear that the total cross section obtained by multiplying (7.12) by the number of black hole micro-states, $n_{\mathrm{st}}=\mathrm{e}^{S_{\mathrm{BH}}}$, saturates unitarity.

In order to keep it sharp: in this discussion, we do not pretend to understand the microscopic origin of $S_{\mathrm{BH}}$. Instead, we simply take it for granted and observe that the structure of the $2 \rightarrow n$ graviton amplitude matches what is expected from a black hole. We are not going further than this. However, a complete similarity with the properties of a non-gravitational saturon bubble - where we do understand the microscopic origin of the entropy - must ring some bell.

The above concludes our check list. It is obvious from this list that we are dealing with striking similarities between two types of objects. On one side, this are saturons in a simple renormalizable theory. Their microscopic properties are as transparent as they could be for a multi-particle state at weak coupling. On the other side, we have black holes in a non-renormalizable theory. Yet, we see that essentially all known properties match. As we have seen, the central source that defines these similarities is that both saturate the bound (1.4). While the reader can decide for themselves how seriously to take this connection, our view is the following:

We think that there is something fundamental about the connection between saturations of unitarity and entropy encoded in the bounds (1.1) and (1.2). This connection goes well beyond gravity or renormalizability. It is the saturation point (1.4) that determines the behaviour of the system, including its decay pattern, life-time, as well as the capacities of information storage and processing.

\section{Saturons and classicalization}

Few years ago [4] it has been suggested that certain theories - that lack sensible Wilsonian UV-completions - can instead be UV-completed by classicalization. The key idea is as follows. Consider a theory in which a coupling $\alpha(q)$ becomes strong above certain cutoff $\Lambda_{U V}$. In such a theory the processes with momentum-transfer $q>\Lambda_{U V}$ are out of control. In certain cases the theory allows to be UV-completed above the scale $\Lambda_{U V}$ by integrating-in new weakly-interacting degrees of freedom. These new degrees of freedom 
restore perturbative unitarity in processes with momentum-transfer $q \gg \Lambda_{U V}$. We call such UV-completion Wilsonian. A nice example of this is the Higgs in the Standard Model which restores unitarity in scattering of longitudinal $W$-bosons at high $q$. What happens when the sensible Wilsonian UV-completion is not possible?

The idea of classicalization is that in such a case the theory can use its classical objects for UV-completion. A classical object of mass $M \gg \Lambda_{U V}$ and size $R \gg \Lambda_{U V}^{-1}$ is composed out of many soft quanta of momenta $q \sim R^{-1} \ll \Lambda_{U V}$. Since $q$ is below the cutoff, the coupling is weak, $\alpha(q) \ll 1$. In this way, a would-be strong coupling is traded for a high multiplicity. Such a classical object represents a coherent state of the sort (2.9) with occupation number $n \sim \alpha(q)^{-1}$.

Now, imagine that a scattering process at center of mass energy $E \sim M \gg \Lambda_{U V}$ is dominated by production of a classical state. Such objects in [4] were referred to as classicalons. In such a case, the momentum-transfer in the process will be $q \ll \Lambda_{U V}$. This is because the constituent quanta of the classical objects are soft. Then, the process, despite being conducted at energy much higher than the cutoff, never probes distances shorter than $R$. So the theory shields itself from the strong coupling regime by becoming effectively-classical.

However, there is a tradeoff: the occupation number must be very high. Correspondingly, the theory must find a way of compensating the exponential suppression of the cross section (3.4). As explained in [22], this requires that the entropy of the classical object is high. Thus, in the language of present discussion, the classicalons must be saturons. Then, from the results of the present paper it follows that for classicalization to work, the following two conditions must be satisfied:

- The theory must contain saturons (classicalons);

- Saturons must form an almost continuous spectrum for $M>\Lambda_{U V}$.

The second requirement comes from our previous findings that each saturon dominates the cross section only in a very narrow window of center of mass energy given by (5.6). Therefore, a theory that is UV-completed by classicalization must deliver a saturon for each value of the center of mass energy.

In a renormalizable asymptotically-free theory the saturons appear with very specific masses (3.10) and sizes (3.11). These are determined by the scale $q$ at which the running 't Hooft coupling reaches the critical value (5.3). So, such a theory cannot be UV-completed by classicalization. But, also there is no need for this since asymptotic-freedom takes care of UV-physics.

On the other hand, non-renormalizable theories can offer a continuous spectrum of saturons in UV. The example of this is gravity. There saturons are black holes. This is why gravity can be unitarized by black hole creation. In fact, the proposal of UV-completion by classicalization [4] was based on a similar proposal for gravity [45].

Now, in order to avoid misunderstanding we must stress that unitarization by black holes works for center of mass energies above the Planck scale $M_{P}$. In fact, higher the better. For processes with the center of mass energies $M_{P}$ in which the momentum transfer is also of order $M_{P}$, the coupling $\alpha_{\text {gr }}$ is order one. The resulting resonances produced in 
such collisions represent micro black holes. These cannot be described classically. This is similar to production of QCD resonances around $\Lambda_{Q C D}$ scale. In the language of [6, 7], they are described as states with $n \sim 1$.

\section{Gravitational species bound}

It has been shown [46-48] that black hole physics puts the following bound on the number of particle species,

$$
\Lambda_{U V} \lesssim \frac{M_{P}}{\sqrt{N}}
$$

Here $\Lambda_{U V}$ represents the scale above which the quantum gravity enters the strong coupling regime to which the semi-classical treatment does not apply.

Equation (9.1) is supported by several argument which can be found in [46-48] and will not be repeated here. We just note that perhaps the physically most transparent one is the following: a black hole of radius smaller than $\Lambda_{U V}^{-1}$ has no way to sustain Hawking's thermal evaporation self-consistently. Now, since Hawking's derivation is exact in semi-classical limit, its invalidity implies a breakdown of semi-classical gravity. Hence, the bound (9.1).

Because it relies exclusively on the validity of well-understood properties of semiclassical black holes, the bound (9.1) is fully non-perturbative. The question therefore is whether this bound can be understood in the language of scattering amplitudes.

The present discussion about the entropy saturation and unitarity answers this question. The relevant processes are the $2 \rightarrow n$ processes in which two initial gravitons produce $n$ particles of momenta $q \sim \frac{M_{P}}{\sqrt{N}}$. The example is depicted on figure 3 . Of course, the final state quanta gravitate and must be properly dresses by infrared gravitons. This standard dressing is independent of entropy of species and is assumed to be done. Again, as before, by power of large- $N$ physics, all non-exponential and non-factorial dependences on $N$ play little role in determining the saturation point. Such factors therefore will be set to one.

Now, the $n$ final-state particles can belong to $N$ different species and Einstein gravity couples to all of them democratically. Due to this, the number of final states is exponentially large. The counting is identical to the one given for a gauge theory with a minor difference in a final degeneracy factor. We shall display the cross section for

$$
n=\frac{1}{\alpha_{\mathrm{gr}}(q)}
$$

where, $\alpha_{\mathrm{gr}}(q)$ is the gravitational coupling given by (7.1). Defining the gravitational analog of the 't Hooft coupling,

$$
\lambda_{\mathrm{gr}} \equiv \alpha_{\mathrm{gr}} N=\frac{q^{2} N}{M_{P}^{2}},
$$

we can write the cross section in the form

$$
\sigma=\left(\mathrm{e}^{-1}\left(1+2 \lambda_{\mathrm{gr}}\right)^{\frac{1}{2}}\left(1+\frac{1}{2 \lambda_{\mathrm{gr}}}\right)^{\lambda_{\mathrm{gr}}}\right)^{\frac{1}{\alpha_{\mathrm{gr}}}} .
$$



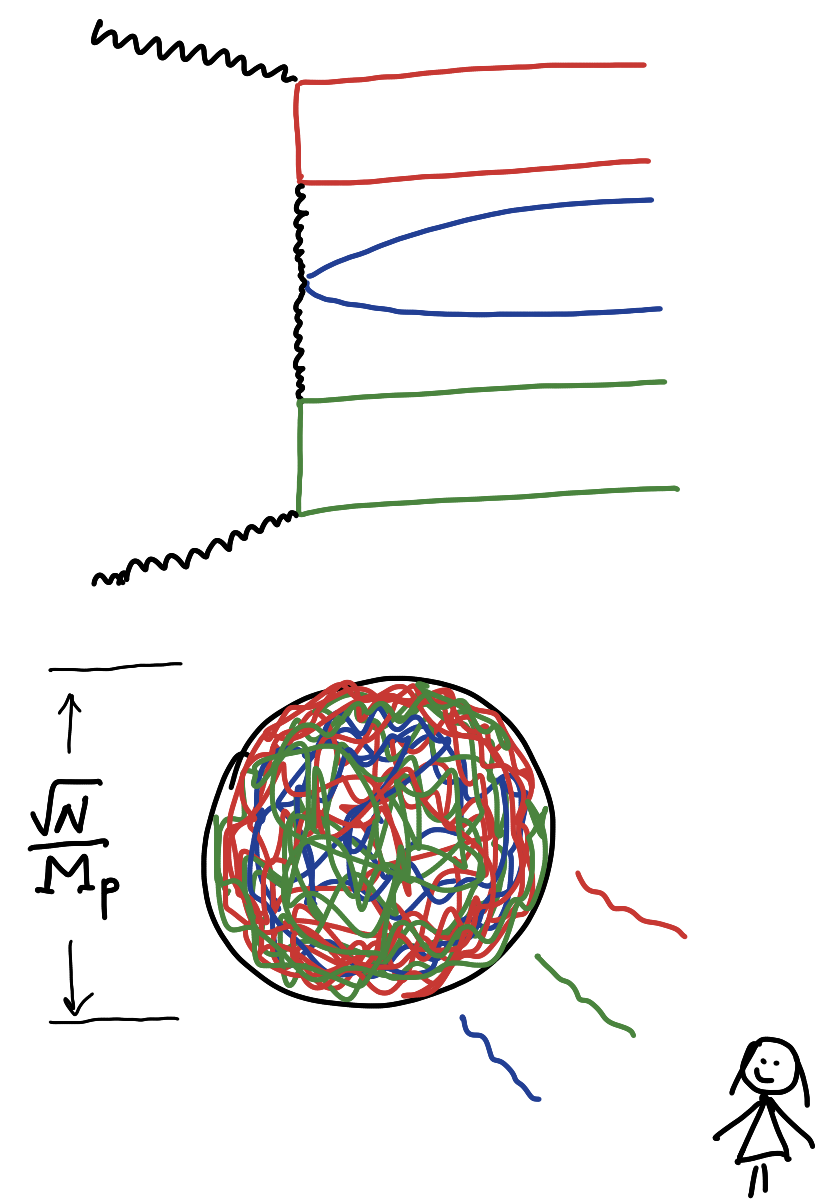

Figure 3. Above, $2 \rightarrow n$ process in which two initial gravitons produce $n$ particles of different species denoted by different colors. The process saturates unitarity at the species scale $\Lambda_{U V} \sim \frac{M_{P}}{\sqrt{N}}$. Below, Alice observing a smallest semi-classical black hole which carries the species hair (denoted by colors).

This cross section saturates unitarity for,

$$
\lambda_{\mathrm{gr}} \simeq 1.1
$$

Obviously, the corresponding value of momentum-transfer $q=\frac{M_{P}}{\sqrt{N}}$ marks the upper bound on UV-cutoff of the theory. It is clear that this bound is exactly the same as the species bound (9.1).

We thus learn that the physical meaning of the species scale $\Lambda_{U V}$ is the following. It determines the value of momentum-transfer $q$ that brings the gravitational 't Hooft coupling to the saturation point (9.5). For this value, the $n$-particle state becomes a saturon. That is, it saturates both the entropy bound and unitarity. This saturon has a very clear physical meaning. It represents a smallest possible semi-classical black hole. Such a black hole carries a species hair [49]. Notice that the entropy derived due to micro-state degeneracy of species, exactly matches the Bekenstein entropy of such a black hole. 


\section{Outlook}

In the present paper we have further explored the ideas about the connection between entropy and unitarity that were introduced in $[1,2]$. The central message is that unitarity of the scattering amplitudes imposes two universal bounds on the entropy of a quantum system. Namely, the maximal entropy is given by the area measured in units of a decay constant $f$ of a relevant Goldstone degree of freedom (1.1). At the same time, the entropy bound is set by the inverse running coupling $\alpha^{-1}$ evaluated at the scale of the size of the object (1.2). These bounds turn out to be more stringent and more general than Bekenstein's classic bound (1.3). In particular, they may be violated by the objects that respect the latter bound. Of course, such systems are eliminated by unitarity. Also, since these bounds have no explicit reference to the energy, they are applicable to the Euclidean entities such as instantons for which the Bekenstein bound cannot be defined. On the other hand, the objects that saturate/respect (1.1) and (1.2) also saturate/respect the Bekenstein bound (1.3). That is, in a consistent theory all three bounds are saturated simultaneously. We refer to the objects that reach this point as saturons.

We have seen that the saturation of both bounds (1.1) and (1.2) is mapped on the saturation of unitarity by $2 \rightarrow n$ scattering amplitudes with $n=\frac{1}{\alpha}$. This saturation is non-perturbative. Naturally, such processes are interpreted as the production of a saturon in two-particle collision.

Now, the saturon is a multi-particle state which is approximately-classical. It therefore appears to defy the standard field theoretic intuition that a production of a classical object in a two-particle collision must be exponentially suppressed. We have explained what is going on in reality. Fist, refining the analysis of [22], we gave a general argument showing that the transition to each individual final state is indeed exponentially suppressed. This is in full accordance with the previous studies [15]-[23]. However, in case of a saturon the suppression is compensated by the exponentially large number of micro-states that are classically-indistinguishable. In other words, the cross section is enhanced due to the entropy of the final state. Due to this, with a properly chosen center of mass energy, the saturon cross section can dominate the scattering process. However, the cross section is very narrowly peaked at a resonant value of the initial energy. Away from this value the cross section diminishes exponentially steeply.

Due to the above properties, saturons can play the role in UV-completion by classicalization [4], but only if they form a continuous spectrum above certain energy. However, it is unclear how wide is the range of such theories.

We have observed that consistent theories dynamically resist to violations of the entropy bounds. An especially interesting example is provided by $\mathrm{SU}(N)$ gauge theory. It was already shown in [2] that an isolated instanton saturates the entropy bounds (1.1) and (1.2) at the critical value of 't Hooft coupling of order one. We have seen that any further increase of the running 't Hooft coupling would violate the entropy bounds. Correspondingly, the scattering amplitudes would violate unitarity. In order to prevent this from happening, the theory must become confining. This puts the phenomenon of confinement in a new light. Namely, it appears that in $\mathrm{SU}(N)$ with pure glue the confinement represents a necessary response that avoids the violations of the entropy bounds and unitarity. In 
other words, in order not to violate the entropy bounds (1.1) and (1.2) somewhere in deep IR, the theory must eliminate the asymptotic colored states. The possible alternatives would be that the theory either hits an IR fixed point or develops a mass gap via the Higgs effect. However, none of the two options are feasible in pure glue. Thus, confinement emerges as a direct consequence of the entropy bounds and unitarity.

Likewise, in [1] it was observed that a baryon saturates the above entropy bounds when the numbers of flavors and colors are of the same order. At this point the baryon entropy satisfies the relation (1.4). The violation of the entropy bounds would render the theory asymptotically non-free. Simultaneously, the multi-pion scattering amplitudes would violate unitarity.

Next, we have constructed an explicit theory that contains saturons. We deliberately chose the example that is maximally distant from gravity. In particular, the theory is renormalizable and not based on any gauge symmetry. The saturons there represent the vacuum bubbles that house a large number of Goldstone modes in their interior. These gapless Goldstone excitations create an exponentially large number of the bubble microstates. The resulting micro-state entropy saturates the bounds (1.1) and (1.2) for a critical value of 't Hooft coupling. At this point, the bubble becomes a saturon.

We have shown that on all counts the bubble saturons behave like black holes. It is also clear that these properties are universal. They must be shared by saturons in other renomalizable theories. The generalization of the constructions given in $[1,2]$ and in the present paper is straightforward. In particular, for making contact with decaying black holes, we need to construct saturons without any net conserved topological charge. The vacuum bubble saturons discussed in this paper have this property. The construction can easily be generalized by creating saturons using pairs of topological or non-topological solitons with opposite charges that are placed on top of one another. For example, one can pair up baryon-antibaryon (skyrmion-anti-skyrmion), monopole-anti-monopole and so on. The annihilation of topological defect has been studied previously numerically. For example, monopole-antimonopole pairs were analysed in [54]. However, to our knowledge, no studies have been done either for the saturated case or in the limit (7.6). The oscillating lumps of the scalar fields, the so-called oscillons [50-52], ${ }^{1}$ can also be used as the building block for constructing a saturon. However, one has to be careful to stay within the regime of weak coupling $\alpha$.

A profound question for future studies is whether there are any implications of the present results for AdS/CFT correspondence [55-57]. Perhaps a natural avenue to go would be to ask whether AdS can be viewed as a saturated state of some gravitational degrees of freedom, as it was suggested in $[6,7]$. No real progress in this direction has been achieved so far. Surprisingly, the analogous approach to de Sitter space turned out to be more straightforward. In particular, the resolution of de Sitter patch in form of a saturated coherent state of gravitons has been discussed in [33, 34].

Finally, our studies bring us to the point at which the properties of a black hole can be understood through the prism of a fundamental connection between unitarity and entropy. We observe that this connection is universal and is shared by saturons irrespective of their origin. This strongly suggest that black hole is a saturon state of gravitons, as was originally proposed in $[6,7]$.

\footnotetext{
${ }^{1}$ For implications for dark matter, see ref. [53].
} 


\section{Acknowledgments}

We thank Akaki Rusetsky and Goran Senjanovic for valuable discussions and comments. This work was supported in part by the Humboldt Foundation under Humboldt Professorship Award, by the Deutsche Forschungsgemeinschaft (DFG, German Research Foundation) under Germany's Excellence Strategy - EXC-2111 — 390814868, and Germany's Excellence Strategy under Excellence Cluster Origins.

It is a great pleasure to thank Lorentz Institute and University of Leiden for hospitality during the stay under the 2020 Lorentz Professorship.

\section{A Argument from effective $S$-matrix}

We shall now give a fully non-perturbative consistency argument explaining why a properly resummed matrix element of transition

$$
\mid \text { few }\rangle \rightarrow \mid \text { many }\rangle
$$

must be exponentially suppressed. This argument is a refined version of the one in [22] and is based on effective $\hat{S}$-matrix. Consider a process describing a transition between two sorts of quanta, denoted by $a$ and $b$ respectively. During it, $l$ particles of species $b$ get converted into $n$ particles of species $a$. Here, the term species specifies all quantum numbers. For, example $a$ and $b$ can denote the different momentum modes of the same quantum field, or some modes of two distinct fields.

We assume that number eigenstates of $a$ and $b$ species represent the legitimate $S$-matrix states over the time-scales of interest. Among other things, this implies that the effective Hamiltonian is approximately diagonal in $a$ and $b$ modes throughout the transition process. That is, the off-diagonal terms in the Hamiltonian must be subleading as compared to the diagonal ones during the relevant time-evolution. This is a necessary condition for having a well-posed transition process. It of course implies that the underlying field theory stays within the weak-coupling regime throughout the transition. The theory shall be otherwise unspecified.

We focus on the case when the occupation number $n$ in the final state is much larger then the analogous number $l$ in the initial state, $\frac{n}{l} \gg 1$. As we shall see, in such a case, the transition matrix element is always exponentially suppressed. This is in accordance with [21]. Therefore, for simplicity we first take $l=1$.

Thus, the initial state is a one-particle state $|i n\rangle=|1\rangle_{b} \otimes|0\rangle_{a}$ with a single $b$-quantum present. Respectively, the final state $|f\rangle=|0\rangle_{b} \otimes|n\rangle_{a}$ is populated with $n$ a-quanta. Of course, we assume that the transition is kinematically allowed.

Now, consider a fully resummed $\hat{S}$-matrix operator. The term that is responsible for the above transition has the form

$$
\hat{S}_{1_{b} \rightarrow n_{a}}=\kappa\left(\hat{a}^{\dagger}\right)^{n} \hat{b}
$$

The form is unique since the operator has to destroy a single particle of species $b$ and create $n$ particles of species $a$. Of course, the operator (A.2) is a result of resummation of infinite 
series. The information about this resummation is contained in the coefficient $\kappa$. We shall now argue that by consistency $\kappa$ is bounded as,

$$
\kappa \lesssim n^{-\frac{n}{2}}
$$

This upper bound is universal and independent of the details of underlying field theory. It may come as a surprise because, naively, all we need to require is that the matrix element satisfies,

$$
\left|\left\langle f\left|\hat{S}_{1_{b} \rightarrow n_{a}}\right| i n\right\rangle\right|^{2}<1 .
$$

The latter requirement would give a much milder bound,

$$
\kappa<\frac{1}{\sqrt{n !}} .
$$

However, the correct bound is (A.3). Here is why: in order to have a well-posed scattering problem, we must demand that the matrix element $\left\langle\psi\left|\hat{S}_{b \rightarrow n a}\right| \psi\right\rangle$ is small over all the states $|\psi\rangle$ that are physically close to either $|i n\rangle$ or $|f\rangle$. The meaning of this requirement we shall now explain.

We define the two normalized states $|1\rangle$ and $|2\rangle$ as physically close if they provide comparable expectation values for a physical observable $\hat{O}$,

$$
\langle 1|\hat{O}| 1\rangle \sim\langle 2|\hat{O}| 2\rangle .
$$

Under comparable we mean the same order of magnitude. The role of the physical observable $\hat{O}$ can be played by an arbitrary measurable quantity. We choose it to be the number operator of $a$-quanta $\hat{n} \equiv \hat{a}^{\dagger} \hat{a}$. The reader should feel free to explore other choices. Then, according to above definition, a state $|\psi\rangle$ is physically close if, for example, $\langle\psi|\hat{n}| \psi\rangle \sim\langle f|\hat{n}| f\rangle$. Our criterion is that on any such state $|\psi\rangle$ the expectation value of $\hat{S}_{b \rightarrow n a}$ must be small. Why?

Here is one way to explain this. Think of the above transition process in terms of timeevolution in the Hilbert space. Let the state vector at some initial time be $|t=0\rangle=\mid$ in $\rangle$. After a sufficiently long time $t$ this state evolves into $|t\rangle$. The projection of $\langle f \| t=\infty\rangle$ determines the $S$-matrix elements. During the time evolution in any given process the state vector explores only a finite portion of the infinite Hilbert space. With the states populating this portion, vector $|t\rangle$ has a significant overlap. These are states that are physically-close to $|t\rangle$. Our requirement then is equivalent to demanding that on all such states the off-diagonal part of the effective Hamiltonian must be smaller than the diagonal part. A violation of this requirement would imply that somewhere in the transition process $a$ and $b$-modes stop to be the valid weakly-coupled degrees of freedom. The Hamiltonian then must be re-diagonalized by a large canonical transformation. This would contradict to our starting point.

Since the state $|\psi\rangle$ can be chosen arbitrarily, we take it to be the following coherent state,

$$
|\psi\rangle=\mathrm{e}^{\sqrt{n}\left(\hat{a}^{\dagger}-\hat{a}\right)+\left(\hat{b}^{\dagger}-\hat{b}\right)}|0\rangle .
$$

Obviously, this state satisfies the criterion of the physical closeness since

$$
\langle\psi|\hat{n}| \psi\rangle=n=\langle f|\hat{n}| f\rangle \text {. }
$$


Therefore, we must require,

$$
\left|\left\langle\psi\left|\hat{S}_{1_{b} \rightarrow n_{a}}\right| \psi\right\rangle\right|^{2}<1
$$

which immediately gives (A.3). Taking this into account, we get

$$
\left|\left\langle f\left|\hat{S}_{1_{b} \rightarrow n_{a}}\right| i n\right\rangle\right|^{2}<n ! n^{-n} \sim \mathrm{e}^{-n},
$$

where in the last step we used Stirling's approximation. Thus, a transition matrix element, describing the creation of any $n$-particle state $|f\rangle$ from a one-particle initial state $|i n\rangle$, must be exponentially suppressed. This is a non-perturbative result. This conclusion is of course in full agreement with the previous studies [15]-[23]. However, it makes the origin of the suppression transparent from very general perspective of $S$-matrix consistency.

Obviously, the above reasoning can be easily generalized to the case in which the occupation number of $b$-particles in the initial state $|i n\rangle=|l\rangle_{b} \otimes|0\rangle_{a}$ is larger than one. As long as the difference between the occupation numbers in initial and final states is large, $n \gg l$, the exponential suppression of the transition matrix element takes place.

We shall now move to the case in which the final particles can belong to several different species. That is, we allow the operators $\hat{a}_{j}$ to carry a species label $j=1,2, \ldots, N$. This label can represent an arbitrary quantum number such as "color" or "flavor". Thus, we are looking for a transition matrix element between an initial state $|i n\rangle=|1\rangle_{b} \otimes|0\rangle_{a}$ and a final state $|f\rangle=|0\rangle_{b} \otimes\left|n_{1}, n_{2}, \ldots n_{N}\right\rangle_{a}$, where $\left|n_{1}, n_{2}, \ldots n_{N}\right\rangle_{a}=\prod_{j=1}^{N} \frac{\left(\hat{a}_{j}^{\dagger}\right)^{n_{j}}}{\sqrt{n_{j} !}}|0\rangle_{a}$, with $\sum_{j=1}^{N} n_{j}=n$. The occupation numbers $n_{j}$ are otherwise unconstrained. That is, the final state $|f\rangle$ houses $n$-quanta with arbitrary color indexes. Of course, when only one color is occupied, the story reduces to the case of singe $a$-species.

Correspondingly, the transition $\hat{S}$-operator now has a form,

$$
\hat{S}_{b \rightarrow n a}=\kappa \prod_{j=1}^{N}\left(\hat{a}_{j}^{\dagger}\right)^{n_{j}} \hat{b},
$$

with the constraint $\sum_{j=1}^{N} n_{j}=n$.

In order to derive an upper bound on the coefficient $\kappa$, we shall repeat the previous reasoning. Namely, we demand a relative smallness of the expectation values of $\hat{S}$ over all the states $|\psi\rangle$ that are physically close to $|f\rangle$. Again, as a test observable we use the total number operator of $a$-species, $\hat{n} \equiv \sum_{j=1}^{N} \hat{a}_{j}^{\dagger} \hat{a}_{j}$. Correspondingly, for $|\psi\rangle$, we use a simple generalization of the state (A.7) to several species,

$$
|\psi\rangle=\mathrm{e}^{\sum_{j=1}^{N} \sqrt{\tilde{n}_{j}}\left(\hat{a}_{j}^{\dagger}-\hat{a}_{j}\right)}|0\rangle .
$$

Here, we have introduced a notation tilde in order to distinguish between the coherent state parameters $\tilde{n}_{j}$ and the corresponding number eigenvalues $n_{j}$. We shall take $\tilde{n}_{j} \sim n_{j}$. Then,

$$
\langle\psi|\hat{n}| \psi\rangle=\sum_{j} \tilde{n}_{j} \sim n=\langle f|\hat{n}| f\rangle
$$

which ensures that the states $|\psi\rangle$ and $|f\rangle$ are physically close. 
Now, demanding the smallness of the expectation value (A.9) evaluated for the $\hat{S}$ matrix operator (A.11) over the coherent state (A.12), we conclude that the coefficient $\kappa$ must obey,

$$
\kappa<\prod_{j=1}^{N} \tilde{n}_{j}^{-\frac{n_{j}}{2}} .
$$

We shall now consider the cases of large and small values of $n_{j}$ separately. We must remember that $n_{j}$-s are characteristics of the transition process, whereas $\tilde{n}_{j}$ are parameters of the probe state $|\psi\rangle$. The latter can be chosen at our convenience subject to $\tilde{n}_{j} \sim n_{j}$.

Now, for the case of large $n_{j}$-s, we can simply take $\tilde{n}_{j}=n_{j}$ and use Stirling approximation in (A.14). Then, for the transition matrix element we get,

$$
\left|\left\langle f\left|\hat{S}_{1_{b} \rightarrow n_{a}}\right| i n\right\rangle\right|^{2}<\prod_{j} n_{j} ! n_{j}^{-n_{j}} \sim \mathrm{e}^{-\sum_{j} n_{j}}=\mathrm{e}^{-n} .
$$

Regarding the case of $n_{j} \sim 1$, it suffices to take $\tilde{n}_{j}$ slightly larger than $n_{j}$. For example, consider the case $n_{j}=1$ for all $j$. Of course, in this case $n=N$. Taking $\tilde{n}_{j}=e n_{j}=e$, we see from (A.14) that the transition matrix element is suppressed as $\mathrm{e}^{-n}=\mathrm{e}^{-N}$.

In summary, we arrive to the universal suppression of a transition matrix element,

$$
\mid\left.\langle\text { many }|\hat{S}| \text { few }\rangle\right|^{2} \lesssim e^{-(\text {many })} .
$$

Here many $=n$ denotes the total occupation number in the final state. This result fully matches the physical intuition which tells us that the creation of classical states in collisions of few quanta must be strongly suppressed. Indeed, the transition $\mid$ few $\rangle \rightarrow \mid$ many $\rangle$ represents a quantum-to-classical transition. The classicality of the final state is obvious when the occupation numbers of the individual species, $n_{j}$, are large. However, the same is also true when the individual numbers $n_{j}$ are small, as long as the total occupation number $n$ is large and coupling $\alpha$ is sufficiently weak. The reason is that the species are only distinguished by the quantum number $j$ that is associated with the weak coupling.

To reiterate, if $n$ is large, the state $|f\rangle$ is essentially classical, even if the individual occupation numbers are minimal, $n_{j}=1$. This is because an observer (Alice) needs a very long time in order to distinguish the individual "colors" of the constituents if their quantum coupling $\alpha$ is extremely weak, $\alpha=\frac{1}{N}$. Indeed, imagine that Alice wishes to distinguish the state $|f\rangle$ with $n_{1}=N, n_{j \neq 1}=0$ from the one with $n_{1}=n_{2}=\ldots=n_{N}=1$. In order to read-out the color content of the state $|f\rangle$, Alice has to initiate an act of interaction between the individual $a$-quanta and some color-sensitive external probe. However, the rate of such interaction is suppressed by powers of $\alpha$. Correspondingly, the minimal timescale required for the measurement per particle is $t \propto \frac{1}{\alpha}$. Thus, the detection of the species quantum identities demands an investment of a macroscopically-long time-scale. On the shorter time-scales, the only observable effects are the collective $N$-particle processes that are controlled by the 't Hooft coupling $\lambda_{t}=\alpha N$. The latter effects do not vanish in the 't Hooft's large- $N$ limit, and therefore, are classically-observable.

Open Access. This article is distributed under the terms of the Creative Commons Attribution License (CC-BY 4.0), which permits any use, distribution and reproduction in any medium, provided the original author(s) and source are credited. 


\section{References}

[1] G. Dvali, Area law saturation of entropy bound from perturbative unitarity in renormalizable theories, Fortsch. Phys. 69 (2021) 2000090 [arXiv: 1906.03530] [INSPIRE].

[2] G. Dvali, Unitarity entropy bound: solitons and instantons, Fortsch. Phys. 69 (2021) 2000091 [arXiv: 1907.07332] [INSPIRE].

[3] J.D. Bekenstein, A universal upper bound on the entropy to energy ratio for bounded systems, Phys. Rev. D 23 (1981) 287 [InSPIRE].

[4] G. Dvali, G.F. Giudice, C. Gomez and A. Kehagias, UV-completion by classicalization, JHEP 08 (2011) 108 [arXiv:1010.1415] [INSPIRE].

[5] J.D. Bekenstein, Black holes and entropy, Phys. Rev. D 7 (1973) 2333 [INSPIRE].

[6] G. Dvali and C. Gomez, Black hole's quantum N-portrait, Fortsch. Phys. 61 (2013) 742 [arXiv: 1112.3359] [INSPIRE].

[7] G. Dvali and C. Gomez, Black holes as critical point of quantum phase transition, Eur. Phys. J. C 74 (2014) 2752 [arXiv:1207.4059] [InSPIRE].

[8] G. 't Hooft, A planar diagram theory for strong interactions, Nucl. Phys. B 72 (1974) 461 [INSPIRE].

[9] E. Witten, Baryons in the expansion, Nucl. Phys. B 160 (1979) 57 [InSPIRE].

[10] T.H.R. Skyrme, A unified field theory of mesons and baryons, Nucl. Phys. 31 (1962) 556 [INSPIRE].

[11] E. Witten, Global aspects of current algebra, Nucl. Phys. B 223 (1983) 422 [InSPIRE].

[12] E. Witten, Current algebra, baryons, and quark confinement, Nucl. Phys. B 223 (1983) 433 [INSPIRE].

[13] W.E. Caswell, Asymptotic behavior of non-Abelian gauge theories to two loop order, Phys. Rev. Lett. 33 (1974) 244 [INSPIRE].

[14] T. Banks and A. Zaks, On the phase structure of vector-like gauge theories with massless fermions, Nucl. Phys. B 196 (1982) 189 [InSPIRE].

[15] L.S. Brown, Summing tree graphs at threshold, Phys. Rev. D 46 (1992) 4125 [hep-ph/9209203] [INSPIRE].

[16] M.B. Voloshin, Estimate of the onset of nonperturbative particle production at high-energy in a scalar theory, Phys. Lett. B 293 (1992) 389 [INSPIRE].

[17] E.N. Argyres, R.H.P. Kleiss and C.G. Papadopoulos, Amplitude estimates for multi-Higgs production at high-energies, Nucl. Phys. B 391 (1993) 42 [INSPIRE].

[18] A.S. Gorsky and M.B. Voloshin, Nonperturbative production of multiboson states and quantum bubbles, Phys. Rev. D 48 (1993) 3843 [hep-ph/9305219] [INSPIRE].

[19] M.V. Libanov, V.A. Rubakov, D.T. Son and S.V. Troitsky, Exponentiation of multiparticle amplitudes in scalar theories, Phys. Rev. D 50 (1994) 7553 [hep-ph/9407381] [INSPIRE].

[20] M.V. Libanov, D.T. Son and S.V. Troitsky, Exponentiation of multiparticle amplitudes in scalar theories. 2. Universality of the exponent, Phys. Rev. D 52 (1995) 3679 [hep-ph/9503412] [INSPIRE]. 
[21] D.T. Son, Semiclassical approach for multiparticle production in scalar theories, Nucl. Phys. B 477 (1996) 378 [hep-ph/9505338] [INSPIRE].

[22] G. Dvali, Classicalization clearly: quantum transition into states of maximal memory storage capacity, arXiv:1804.06154 [INSPIRE].

[23] A. Monin, Inconsistencies of higgsplosion, arXiv:1808.05810 [INSPIRE].

[24] J.M. Cornwall, On the high-energy behavior of weakly coupled gauge theories, Phys. Lett. B 243 (1990) 271 [INSPIRE].

[25] H. Goldberg, Breakdown of perturbation theory at tree level in theories with scalars, Phys. Lett. B 246 (1990) 445 [INSPIRE].

[26] G. Dvali, D. Flassig, C. Gomez, A. Pritzel and N. Wintergerst, Scrambling in the black hole portrait, Phys. Rev. D 88 (2013) 124041 [arXiv:1307.3458] [INSPIRE].

[27] N.A. Voronov and I.Y. Kobzarev, On three-dimensional periodic solutions of scalar Higgs equation, JETP Lett. 24 (1976) 532 [INSPIRE].

[28] I.L. Bogolyubsky and V.G. Makhankov, On the pulsed soliton lifetime in two classical relativistic theory models, JETP Lett. 24 (1976) 12 [INSPIRE].

[29] T. Belova, N. Voronov, I. Kobzarev and N. Konyukhova, Particle-like solution to the Higgs equation, Sov. Phys. JETP 46 (1977) 846 [Zh. Eksp. Teor. Fiz. 73 (1977) 1611] [InSPIRE].

[30] S.W. Hawking, Particle creation by black holes, Commun. Math. Phys. 43 (1975) 199 [Erratum ibid. 46 (1976) 206] [INSPIRE].

[31] D.N. Page, Information in black hole radiation, Phys. Rev. Lett. 71 (1993) 3743 [hep-th/9306083] [INSPIRE].

[32] G. Dvali, Non-thermal corrections to Hawking radiation versus the information paradox, Fortsch. Phys. 64 (2016) 106 [arXiv:1509.04645] [INSPIRE].

[33] G. Dvali and C. Gomez, Quantum compositeness of gravity: black holes, AdS and inflation, JCAP 01 (2014) 023 [arXiv: 1312.4795] [INSPIRE].

[34] G. Dvali, C. Gomez and S. Zell, Quantum break-time of de Sitter, JCAP 06 (2017) 028 [arXiv: 1701.08776] [INSPIRE].

[35] G. Dvali, A microscopic model of holography: survival by the burden of memory, arXiv: 1810.02336 [INSPIRE].

[36] G. Dvali, L. Eisemann, M. Michel and S. Zell, Universe's primordial quantum memories, JCAP 03 (2019) 010 [arXiv: 1812.08749] [INSPIRE].

[37] G. 't Hooft, Graviton dominance in ultrahigh-energy scattering, Phys. Lett. B 198 (1987) 61 [INSPIRE].

[38] D. Amati, M. Ciafaloni and G. Veneziano, Superstring collisions at Planckian energies, Phys. Lett. B 197 (1987) 81 [inSPIRE].

[39] D. Amati, M. Ciafaloni and G. Veneziano, Classical and quantum gravity effects from Planckian energy superstring collisions, Int. J. Mod. Phys. A 3 (1988) 1615 [INSPIRE].

[40] D.J. Gross and P.F. Mende, The high-energy behavior of string scattering amplitudes, Phys. Lett. B 197 (1987) 129 [INSPIRE].

[41] D.J. Gross and P.F. Mende, String theory beyond the Planck scale, Nucl. Phys. B 303 (1988) 407 [INSPIRE]. 
[42] G. Dvali, C. Gomez and A. Kehagias, Classicalization of gravitons and Goldstones, JHEP 11 (2011) 070 [arXiv: 1103.5963] [InSPIRE].

[43] G. Dvali, C. Gomez, R.S. Isermann, D. Lüst and S. Stieberger, Black hole formation and classicalization in ultra-Planckian $2 \rightarrow N$ scattering, Nucl. Phys. B 893 (2015) 187 [arXiv: 1409.7405] [INSPIRE].

[44] A. Addazi, M. Bianchi and G. Veneziano, Glimpses of black hole formation/evaporation in highly inelastic, ultra-planckian string collisions, JHEP 02 (2017) 111 [arXiv:1611.03643] [INSPIRE].

[45] G. Dvali and C. Gomez, Self-completeness of Einstein gravity, arXiv:1005.3497 [INSPIRE].

[46] G. Dvali, Black holes and large $N$ species solution to the hierarchy problem, Fortsch. Phys. 58 (2010) 528 [arXiv:0706.2050] [INSPIRE].

[47] G. Dvali and M. Redi, Black hole bound on the number of species and quantum gravity at LHC, Phys. Rev. D 77 (2008) 045027 [arXiv: 0710.4344] [InSPIRE].

[48] G. Dvali and C. Gomez, Quantum information and gravity cutoff in theories with species, Phys. Lett. B 674 (2009) 303 [arXiv:0812.1940] [INSPIRE].

[49] G. Dvali, Nature of microscopic black holes and gravity in theories with particle species, Int. J. Mod. Phys. A 25 (2010) 602 [arXiv:0806.3801] [InSPIRE].

[50] I.L. Bogolyubsky and V.G. Makhankov, Lifetime of pulsating solitons in some classical models, Pisma Zh. Eksp. Teor. Fiz. 24 (1976) 15 [INSPIRE].

[51] M. Gleiser, Pseudostable bubbles, Phys. Rev. D 49 (1994) 2978 [hep-ph/9308279] [InSPIRE].

[52] E.J. Copeland, M. Gleiser and H.R. Muller, Oscillons: resonant configurations during bubble collapse, Phys. Rev. D 52 (1995) 1920 [hep-ph/9503217] [INSPIRE].

[53] J. Ollé, O. Pujolàs and F. Rompineve, Oscillons and dark matter, JCAP 02 (2020) 006 [arXiv: 1906.06352] [INSPIRE].

[54] T. Vachaspati, Monopole-antimonopole scattering, Phys. Rev. D 93 (2016) 045008 [arXiv: 1511.05095] [INSPIRE].

[55] J.M. Maldacena, The large $N$ limit of superconformal field theories and supergravity, Int. J. Theor. Phys. 38 (1999) 1113 [hep-th/9711200] [INSPIRE].

[56] S.S. Gubser, I.R. Klebanov and A.M. Polyakov, Gauge theory correlators from noncritical string theory, Phys. Lett. B 428 (1998) 105 [hep-th/9802109] [InSPIRE].

[57] E. Witten, Anti-de Sitter space and holography, Adv. Theor. Math. Phys. 2 (1998) 253 [hep-th/9802150] [INSPIRE]. 